\title{
Twenty-Eight-Day Repeated Inhalation Toxicity Study of Nano-Sized Neodymium Oxide in Male Sprague-Dawley Rats
}

\author{
Yong-Soon Kim ', Cheol-Hong Lim ', Seo-Ho Shin ${ }^{1,2}$ and Jong-Choon Kim ${ }^{2}$ \\ 'Chemicals Toxicity Research Bureau, Occupational Safety and Health Research Institute, KOSHA, Daejeon, Korea \\ ${ }^{2}$ College of Veterinary Medicine BK21 Plus Project Team, Chonnam National University, Gwangju, Korea
}

\begin{abstract}
Neodymium is a future-oriented material due to its unique properties, and its use is increasing in various industrial fields worldwide. However, the toxicity caused by repeated exposure to this metal has not been studied in detail thus far. The present study was carried out to investigate the potential inhalation toxicity of nano-sized neodymium oxide $\left(\mathrm{Nd}_{2} \mathrm{O}_{3}\right)$ following a 28-day repeated inhalation exposure in male SpragueDawley rats. Male rats were exposed to nano-sized $\mathrm{Nd}_{2} \mathrm{O}_{3}$-containing aerosols via a nose-only inhalation system at doses of $0 \mathrm{mg} / \mathrm{m}^{3}, 0.5 \mathrm{mg} / \mathrm{m}^{3}, 2.5 \mathrm{mg} / \mathrm{m}^{3}$, and $10 \mathrm{mg} / \mathrm{m}^{3}$ for $6 \mathrm{hr} /$ day, 5 days $/$ week over a 28 -day period, followed by a 28 -day recovery period. During the experimental period, clinical signs, body weight, hematologic parameters, serum biochemical parameters, necropsy findings, organ weight, and histopathological findings were examined; neodymium distribution in the major organs and blood, bronchoalveolar lavage fluid (BALF), and oxidative stress in lung tissues were analyzed. Most of the neodymium was found to be deposited in lung tissues, showing a dose-dependent relationship. Infiltration of inflammatory cells and pulmonary alveolar proteinosis (PAP) were the main observations of lung histopathology. Infiltration of inflammatory cells was observed in the $2.5 \mathrm{mg} / \mathrm{m}^{3}$ and higher dose treatment groups. PAP was observed in all treatment groups accompanied by an increase in lung weight, but was observed to a lesser extent in the $0.5 \mathrm{mg} / \mathrm{m}^{3}$ treatment group. In BALF analysis, total cell counts, including macrophages and neutrophils, lactate dehydrogenase, albumin, interleukin-6, and tumor necrosis factor-alpha, increased significantly in all treatment groups. After a 4-week recovery period, these changes were generally reversed in the $0.5 \mathrm{mg} / \mathrm{m}^{3}$ group, but were exacerbated in the $10 \mathrm{mg} / \mathrm{m}^{3}$ group. The lowest-observed-adverse-effect concentration of nano-sized $\mathrm{Nd}_{2} \mathrm{O}_{3}$ was determined to be $0.5 \mathrm{mg} / \mathrm{m}^{3}$, and the target organ was determined to be the lung, under the present experimental conditions in male rats.
\end{abstract}

Key words: Inhalation toxicity, Nose-only exposure, Nano-sized neodymium oxide, Lung, Lowestobserved-adverse-effect concentration

\section{INTRODUCTION}

Neodymium is one of the rare earth elements (REEs), which include 17 metal elements (15 lanthanides and 2 nonlanthanides) sharing similar physicochemical and biological properties, and is classified as a light REE along with

Correspondence to: Cheol-Hong Lim, Chemicals Toxicity Research Bureau, Occupational Safety and Health Research Institute, KOSHA, Daejeon 34122, Korea

E-mail: limch@kosha.or.kr

This is an Open-Access article distributed under the terms of the Creative Commons Attribution Non-Commercial License (http:// creativecommons.org/licenses/by-nc/3.0) which permits unrestricted non-commercial use, distribution, and reproduction in any medium, provided the original work is properly cited. lanthanum, cerium, and praseodymium (1). Previously, use of REEs was limited as they readily form compounds such as halides, carbonates, oxides, phosphates, and silicates. As separation and refinement technologies have developed, pure REEs can now be obtained and, are thus utilized in various industrial fields owing to their unique physical and chemical properties $(2,3)$. Neodymium is used in permanent magnets, audio systems, hybrid engines, headphones, catalysts, wind turbines, and hard drives in laptops. The NdFe-B magnet, an alloy of neodymium, iron, and boron, is the strongest permanent magnet and is utilized in various information technologies as well as green technologies due to its high efficiency. Demand for neodymium is increasing globally, and annual production is 7,300 tons, accounting for the fourth largest REE production (4). 
However, as the use of REEs increases in modern industry, several occupational health issues have been reported (5-11). These case reports indicated that pulmonary disorders such as pneumoconiosis, dyspnea, and fibrosis were observed among workers who were chronically exposed to fumes or smoke from REE-containing cored carbon arc lamps during photoengraving and projection operations. Notably, Sulotto et al. (8) and Porru et al. (11) reported that levels of REEs increased abnormally in the bronchoalveolar lavage fluids (BALF), nails, and lung tissues of the individuals. Moreover, bioaccumulation in humans and environmental risks can occur during mining, processing, manufacturing, and waste disposal activities, as REEs are produced and then mainly disposed of, rather than recovered or reused (12).

A review of the toxicological profiles for neodymium and its compounds showed that neodymium oxide $\left(\mathrm{Nd}_{2} \mathrm{O}_{3}\right)$ was less toxic because its $\mathrm{LC}_{50}(50 \%$ of the lethal concentration) and $\mathrm{LD}_{50}(50 \%$ of the lethal dose $)$ are $4,980 \mathrm{mg} / \mathrm{m}^{3}$ and $>5,000 \mathrm{mg} / \mathrm{kg}$, respectively, in rats (13). However, toxicity in specific organs was reported in few repeated toxicity studies. After 30 days of repeated oral administration in mice, neodymium chloride $\left(\mathrm{NdCl}_{3}\right)$ inhibited cell and humoral immunity and induced liver toxicity at a dose of $20 \mathrm{mg} / \mathrm{kg}$ body weight (14), and prolonged oral administration of $\mathrm{NdCl}_{3}$ for 60 days at the same dose induced additional kidney and heart toxicity in mice (15). Referring to the liver toxicity of $\mathrm{NdCl}_{3}$ in mice, Huang et al. (16) reported that repeated oral administration caused an accumulation of $\mathrm{NdCl}_{3}$ in the nuclei of hepatocytes and resulted in liver toxicity by inducing oxidative stress. Zhao et al. (17) reported that 14 days of repeated intraperitoneal injection of $20 \mathrm{mg} / \mathrm{kg} \mathrm{NdCl}$ resulted in brain toxicity through induction of oxidative stress in mice. In a different in vitro cytotoxicity study, $\mathrm{Nd}_{2} \mathrm{O}_{3}$ was cytotoxic to rat pulmonary alveolar macrophages, showing an $\mathrm{LC}_{50}$ of $101 \mu \mathrm{M}(18)$. Moreover, in previous in vivo and in vitro studies, as the particle size of REEs decreased, increased toxicity was observed in lung tissue and cell lines (19-21).

Although some human case reports and animal experiments related to neodymium imply occupational health risks, and utilization of neodymium nanomaterial is expected to increase in the automobile and high-tech industries in the near future, regulations or formal management systems in the work environment have not yet been established. Furthermore, long-term inhalation toxicity studies required for extrapolation of data from animals to humans have not been performed thus far. Therefore, as part of a project to provide toxicological data required for the risk/hazard assessment of neodymium, the present study set to qualitatively and quantitatively evaluate the toxicity of nanosized $\mathrm{Nd}_{2} \mathrm{O}_{3}$ after 28 days of repeated inhalation exposure in Sprague-Dawley rats, and to determine gross toxicity, no-observed-adverse-effect concentration, and the target organs.

\section{MATERIALS AND METHODS}

Generation of nano-sized $\mathbf{N d}_{2} \mathbf{O}_{3}$. A single lot (Aldrich634611) of neodymium (III) oxide (purity: 99.9\%) was purchased from Sigma-Aldrich (St. Louis, Mo, USA). The surface area of nano-sized $\mathrm{Nd}_{2} \mathrm{O}_{3}$ used in the present study was measured to be $17.45 \mathrm{~m}^{2} / \mathrm{g}$ using the Brunauer-EmmettTeller (BET) method (ASAP 2420, Micromeritics Inc., Norcross, GA, USA), and the particle size was calculated to be $49.05 \mathrm{~nm}$. Nano-sized $\mathrm{Nd}_{2} \mathrm{O}_{3}$ was suspended in distilled water at a concentration of 0.09 to $1.50 \%$ and sonicated for $30 \mathrm{~min}$ ( $5 \mathrm{~s}$ sonication $/ 3 \mathrm{~s}$ rest cycle) using a probe-type ultrasonicator (VC750, Sonics \& Materials Inc., Newtown, CT, USA). The hydrodynamic diameter was then measured using the dynamic light scattering method (Zetasizer Nano ZS 90, Malvern, UK) to check particle size and dispersity. The dispersed suspension was aerosolized through a 0.6 $0.8 \mathrm{~mm}$ diameter orifice at an airflow of $4 \sim 8 \mathrm{~L} / \mathrm{min}$ in a nose-only inhalation chamber (NITC system, HCT Co., Icheon, Korea) under constant agitation. The total airflow for each chamber was set at $20 \mathrm{~L} / \mathrm{min}$ to achieve a $1.0 \mathrm{~L} / \mathrm{min}$ flow per rat. Temperature, relative humidity, pressure, and air ventilation in the chambers were recorded automatically.

Nano-sized $\mathrm{Nd}_{2} \mathrm{O}_{3}$ analysis and inhalation chamber monitoring. In a preliminary test, suitability of an optical particle counter (OPC, aerosol spectrometer and dust monitor 1.108, GRIMM spectrometer, GA, USA) for measuring the size distribution of nano-sized $\mathrm{Nd}_{2} \mathrm{O}_{3}$ was verified by using it in combination with a scanning mobility particle sizer system (SMPS); an electrical particle sizer (EPS, 4410, HCT Co.), and a condensation particle counter (CPC, 4312, HCT Co.). During the exposure period, the particle size distribution and mass concentration of the aerosols in the chambers were measured once every $2 \mathrm{hr}$ using an OPC. The aerosol samples in the chambers were also collected for analyses by transmission electron microscopy (TEM, H-7100FA, Hitachi, Tokyo, Japan) and inductively coupled plasma mass spectrometry (ICP-MS, 7500CE, Agilent Technologies, Santa Clara, CA, USA), and compared with the results obtained using OPC. The samples were collected from the middle region of the port using an MSAoperated sampling pump (Escort Elf pump, MSA, PA, USA) at a flow rate of $1.0 \mathrm{~L} / \mathrm{min}$, with nitrocellulose membrane filters of $37 \mathrm{~mm}$ in diameter (Millipore, $0.22 \mu \mathrm{m}$, GSWP, County Cork, Ireland). TEM was performed at a magnification of 50,000 $\times$ for the high dose samples, and the particles were analyzed using an energy dispersive X-ray spectrometer (EDS, EX200, Horiba, Kyoto, Japan) at an accelerating voltage of $75 \mathrm{kV}$.

Experimental animals and animal husbandry. Specific pathogen-free Sprague-Dawley rats were used. Seventy six-week-old male rats were purchased from Japan 
SLC Inc. (Tokyo, Japan), and were acclimated for two weeks before initial exposure. The animals were housed in a room maintained at $22 \pm 3^{\circ} \mathrm{C}$ and at a relative humidity of $50 \pm$ 20\% with artificial lighting (150 300 Lux) from 09:00 to 21:00 and 13 18 air changes per hour. No more than four animals were housed in each solid bottom polysulfone cage $(235 \times 380 \times 175 \mathrm{~mm})$ containing sterilized bedding. Animals were provided irradiation-sterilized pellet feed (LabDiet 5053, PMI Nutrition, St. Louis, MO, USA) and UVsterilized and filtered water ad libitum. The study protocol was approved by the Institutional Animal Care and Use Committee of Chemicals Toxicity Research Bureau (IACUC1602).

Study design overview. Dose selection was determined by considering previous repeated inhalation toxicity results for cerium, which possesses similar physiochemical properties to neodymium. Lowest-observed-adverse-effect concentration (LOAEC) of $\mathrm{CeO}_{2}$ (mass median aerodynamic diameter; MMAD: about $2 \mu \mathrm{m}$ ) was $5 \mathrm{mg} / \mathrm{m}^{3}$ after 13 weeks of exposure in rats (13). Therefore, doses of 10, 2.5, and 0.5 $\mathrm{mg} / \mathrm{m}^{3}$ were set as the high, middle, and low doses, respectively, in the present study. Each group consisted of 16 (control, low, and high dose groups) or 15 (middle dose group) rats, and eight rats per group were sacrificed after a 28-day exposure period. The other rats were set as recovery group, and were necropsied after a 28-day recovery period to identify reversibility, persistence, and delayed occurrence of toxic effects. The body weight range of rats was 287.3 $326.0 \mathrm{~g}$ at first exposure. Exposure to nano-sized $\mathrm{Nd}_{2} \mathrm{O}_{3}$ was performed for $6 \mathrm{hr} /$ day, 5 days/week, for 28 days. The following parameters were examined during the experimental period: clinical signs, mean body weight, hematological parameters, serum biochemical parameters, necropsy findings, organ weight, histopathological parameters, BALF, oxidative stress, and neodymium levels in major organs.

Clinical signs and body weight. Clinical signs, including respiratory, dermal, behavioral, nasal, or genitourinary changes, and mortality, were observed for all animals before, during, and after exposure. Any signs related to the exposure were individually recorded. The initial day of exposure was set as Day 1. Body weight was measured using an electronic balance (QUINTIX3102, Sartorius Co., Göttingen, Lower Saxony, Germany) on Day 1 and once per week afterward, during the entire experimental period. The body weight at necropsy was also measured, after an overnight fast.

Hematology and serum biochemistry. Each blood sample (approximately $2 \mathrm{~mL}$ ) was placed into a CBC bottle (Vacutainer $3 \mathrm{~mL}, \mathrm{BD}$, USA) containing the anticoagulant EDTA-2K and analyzed using an automatic hematology analyzer (ADVIA 2120i, Siemens Diagnostics, Tarrytown, NY, USA). Hematology analysis included the following parameters: red blood cells, hemoglobin concentration, hematocrit, mean corpuscular volume, mean corpuscular hemoglobin, mean corpuscular hemoglobin concentration, red cell distribution width, mean platelet volume, platelet count, white blood cells (WBC), and WBC differential count (neutrophils, lymphocytes, monocytes, eosinophils, and basophils). Additional blood samples $(3 \mathrm{~mL})$ were collected at the same time as the hematology samples and placed into $5 \mathrm{~mL}$ vacutainer tubes (BD Pharmingen, San Diego, CA, USA) containing a clot activator. The samples were maintained at room temperature for 15 20 min to allow coagulation, and then centrifuged at 3,000 rpm (LABMASTER ABC-CB200R, HANLAB, Cheongju, Korea) for $10 \mathrm{~min}$ using a serum biochemistry analyzer (TBA-120FR, Toshiba Co., Tokyo, Japan). Serum biochemistry analysis included the following parameters: aspartate aminotransferase, alanine aminotransferase, alkaline phosphatase, gamma glutamyl transferase, creatine phosphokinase, total bilirubin, glucose, total cholesterol, triglycerides, total protein, albumin, blood urea nitrogen, creatinine, inorganic phosphorus, lactate dehydrogenase (LDH), and calcium ions.

Necropsy and organ weight. On the day of scheduled necropsy, all surviving animals were euthanized by isoflurane (Ilsung Pharm. Co., Seoul, Korea) inhalation. Blood was taken from the posterior vena cava for hematology and serum biochemistry analyses after confirming anesthesia. The abdominal aorta and posterior vena cava were cut for exsanguination and sacrifice. Macroscopic examinations were performed for the body surface, subcutis, and all internal organs in the head, abdominal and thoracic cavities, and then the kidneys, spleen, lungs, brain, and liver were removed and weighed with an electronic balance (QUINTIX313, Sartorius Co.): bilateral organs were measured together. Weights were recorded both as absolute organ weight and as relative organ weight. The relative organ weight was calculated based on the ratio of absolute organ weight to fasted body weight.

Histopathology. Microscopic examinations were performed on the weighed organs, including hilar lymph node, left lung, nasal cavity, trachea, and abnormal lesions defined by the study pathologist. The organs and tissues were fixed in $10 \%$ neutral buffered formalin solution. The nasal cavity was sectioned at four levels: posterior to the upper incisors, the incisive papilla, the second palatine ridge, and the first molar teeth. The samples were stained with hematoxylin and eosin, and then examined using a light microscope at 100 or $200 \times$ magnification.

Bronchoalveolar lavage fluid (BALF) analysis. The right lung was lavaged five times with $3 \mathrm{~mL}$ of calcium- and magnesium-free phosphate buffered saline (PBS, pH 7.4) after tracheal cannulation using a PE-90 tube (ClayAdams, 
NJ, USA). The lavaged fluids were centrifuged at 1,500 rpm for $10 \mathrm{~min}$ (Hanil Union 32R, Incheon, Korea). The supernatants of the first lavage fluid were stored at $-80^{\circ} \mathrm{C}$ for subsequent albumin, interleukin-6 (IL-6), tumor necrosis factor-alpha (TNF- $\alpha$ ), and LDH assays. The portion containing the collected BALF pellet was used for total cell counts using an automatic hematology analyzer (ADVIA 2120i, Siemens Diagnostics). The remainder was used for differential leukocyte counts after centrifugation at 1,500 rpm for $10 \mathrm{~min}$ (Hanil Cellspin, Incheon, Korea) and DiffQuick staining (Sysmex, Kobe, Japan). About 300 leukocytes (macrophages, neutrophils, lymphocytes, and eosinophils) were counted under a microscope at $400 \times$ magnification. The albumin and LDH levels in the lavage fluid were measured using a biochemistry analyzer (TBA-120FR, Toshiba Co.), while TNF- $\alpha$ (RTA00, R\&D Systems, Minneapolis, MN, USA) and IL-6 (R6000B, R\&D Systems) levels in the lavage fluid were measured using commercial assay kits.

Oxidative stress analysis. The right lungs of the control and treatment groups were homogenized in five volumes of $50 \mathrm{mM}$ phosphate buffer solution $\left[\mathrm{KH}_{2} \mathrm{PO}_{4}\right.$ (Sigma, P5379), $\mathrm{K}_{2} \mathrm{HPO}_{4}$ (Sigma, P3786), pH 7.0]. Catalase (Cayman catalase kit 707002) and reduced glutathione (GSH) (Cayman glutathione assay kit 703002) were measured in the homogenized tissue, which had been adjusted to a final protein concentration of $2 \mathrm{mg} / \mathrm{mL}$.

Measurement of neodymium levels. Neodymium levels were measured using ICP-MS. The right lung, liver, spleen, kidneys, and brain tissues were digested in five or ten volumes of $69 \%$ nitric acid (Merck, Whitehouse Station, NJ, USA) before analysis. Ten volumes of $69 \%$ nitric acid were used for analysis of whole blood. The analytical conditions of ICP-MS are shown in Table 1.

Statistical analysis. Data are presented as mean \pm standard deviation (SD). Body weight, hematological parame-

Table 1. Neodymium analysis condition of inductively coupled plasma mass spectrometry

\begin{tabular}{llc}
\hline \hline Parameter & Unit & Value \\
\hline RF power & $\mathrm{W}$ & 1,600 \\
Sampling depth & $\mathrm{Mm}$ & 8 \\
Torch-H & $\mathrm{Mm}$ & -0.4 \\
Torch-V & $\mathrm{Mm}$ & 0.4 \\
Carrier gas & $\mathrm{L} / \mathrm{min}$ & 0.7 \\
Makeup gas & $\mathrm{L} / \mathrm{min}$ & 0.5 \\
S/C temp & ${ }^{\circ} \mathrm{C}$ & 2 \\
He gas & $\mathrm{mL} / \mathrm{min}$ & 5 \\
Nd & $\mathrm{m} / \mathrm{z}$ & 146 \\
\hline
\end{tabular}

$\mathrm{RF}$, radio frequency; $\mathrm{He}$, helium; $\mathrm{Nd}$, neodymium; $\mathrm{m} / \mathrm{z}$, mass to charge ratio. ters, serum biochemical parameters, organ weight, BALF and oxidative stress parameters, and neodymium levels were assumed to be normally distributed and analyzed by one-way analysis of variance (ANOVA). Dunnett's T3 test was used as the post hoc test. SPSS $22.0 \mathrm{~K}$ software was used for all statistical analyses (Chicago, IL, USA). A $p$ value $<0.05$ was considered significant.

\section{RESULTS}

Generation of nano-sized $\mathrm{Nd}_{2} \mathrm{O}_{3}$ and inhalation chamber monitoring. To maintain a constant exposure concentration, concentration of dispersion solution and aeration flow within the inhalation chamber were adjusted daily during the exposure period. For each treatment group, characterization of the nano-sized $\mathrm{Nd}_{2} \mathrm{O}_{3}$ dispersion solution during the exposure period is presented in Table 2 . The concentrations of dispersion solution were $0.10 \pm 0.01 \%, 0.40 \pm$ $0.00 \%$, and $1.11 \pm 0.13 \%$ in the low, middle, and high dose groups, respectively. The particle sizes of the dispersion solution were $206 \pm 31.0 \mathrm{~nm}, 267 \pm 14.8 \mathrm{~nm}$, and $273 \pm 13.2$ $\mathrm{nm}$, and the polydispersity indices were $0.23 \pm 0.05,0.25 \pm$ 0.05 , and $0.31 \pm 0.04$, for the low, middle, and high dose groups, respectively, after sonication. During sonication, the applied energies, from low to high dose groups, were $251 \pm$ $33.0 \mathrm{~J} / \mathrm{mL}, 253 \pm 38.8 \mathrm{~J} / \mathrm{mL}$, and $257 \pm 36.5 \mathrm{~J} / \mathrm{mL}$, respectively. For each treatment group, characterization of the nano-sized $\mathrm{Nd}_{2} \mathrm{O}_{3}$ aerosol in the chamber during the exposure period is presented in Table 3 and Fig. 1. The actual concentrations measured using the OPC were $0.51 \pm 0.03$

Table 2. Characterization of nano-sized $\mathrm{Nd}_{2} \mathrm{O}_{3}$ dispersion during the exposure period

\begin{tabular}{lccc}
\hline \hline \multirow{2}{*}{ Parameter } & \multicolumn{3}{c}{ Dose $\left(\mathrm{mg} / \mathrm{m}^{3}\right)$} \\
\cline { 2 - 4 } & \multicolumn{1}{c}{0.5} & \multicolumn{1}{c}{2.5} & \multicolumn{1}{c}{10} \\
\hline Concentration (\%) & $0.10 \pm 0.01^{\mathrm{a}}$ & $0.40 \pm 0.00$ & $1.11 \pm 0.13$ \\
Energy (Joule/mL) & $251 \pm 33.0$ & $253 \pm 38.8$ & $257 \pm 36.5$ \\
Size (nm) & $206 \pm 31.0$ & $267 \pm 14.8$ & $273 \pm 13.2$ \\
PDI & $0.23 \pm 0.05$ & $0.25 \pm 0.05$ & $0.31 \pm 0.04$ \\
\hline
\end{tabular}

PDI, polydispersity index.

aalues are expressed as means \pm SD $(n=20)$.

Table 3. Aerosol concentration and particle size of nano-sized $\mathrm{Nd}_{2} \mathrm{O}_{3}$ in chambers during the exposure period

\begin{tabular}{lccr}
\hline \hline \multirow{2}{*}{ Parameter } & \multicolumn{3}{c}{ Dose $\left(\mathrm{mg} / \mathrm{m}^{3}\right)$} \\
\cline { 2 - 4 } & 0.5 & 2.5 & \multicolumn{1}{c}{10} \\
\hline Concentration $\left(\mathrm{mg} / \mathrm{m}^{3}\right)$ & $0.51 \pm 0.03^{\mathrm{a}}$ & $2.55 \pm 0.07$ & $10.09 \pm 0.18$ \\
MMAD $(\mu \mathrm{m})$ & $0.27 \pm 0.02$ & $0.43 \pm 0.02$ & $0.67 \pm 0.02$ \\
GSD & $1.75 \pm 0.13$ & $1.56 \pm 0.07$ & $1.53 \pm 0.02$ \\
\hline
\end{tabular}

MMAD, mass median aerodynamic diameter; GSD, geometric standard deviation.

${ }^{a}$ Values are expressed as means \pm SD $(n=60)$. 


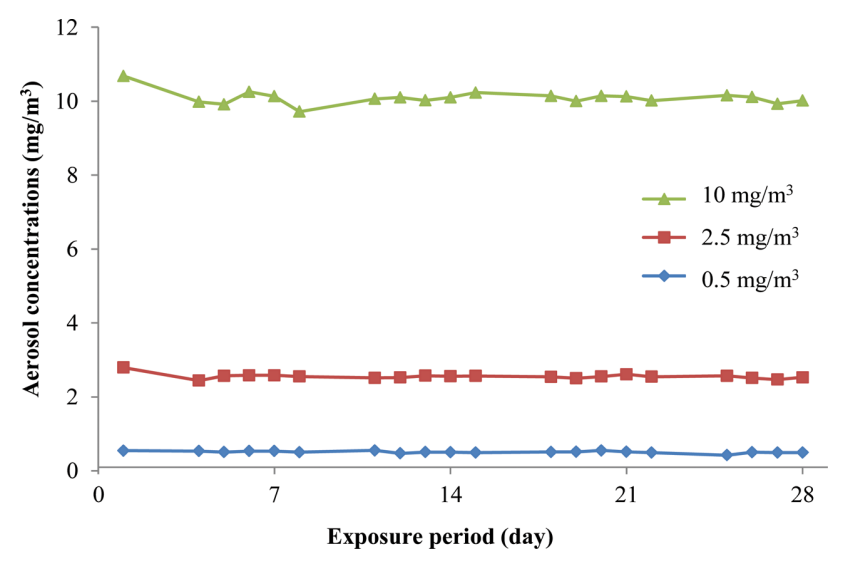

Fig. 1. Aerosol concentrations of nano-sized $\mathrm{Nd}_{2} \mathrm{O}_{3}$ at $0.5(\bullet)$, $2.5(\boldsymbol{\square})$, and $10(\boldsymbol{\Delta}) \mathrm{mg} / \mathrm{m}^{3}$, during the exposure period.
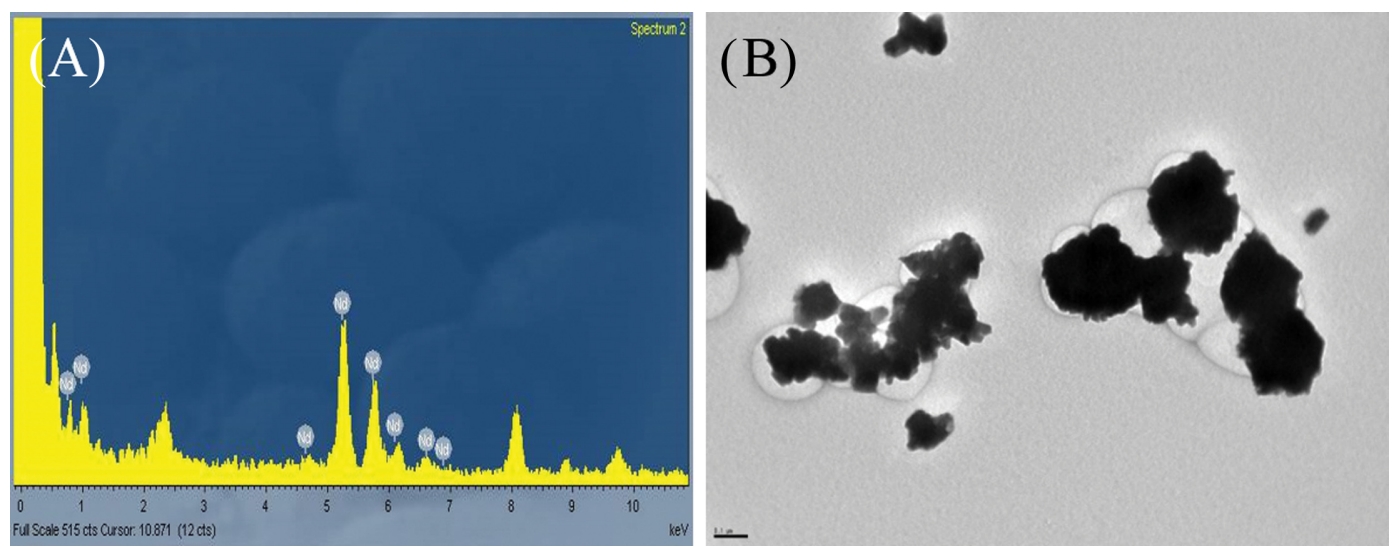

Fig. 2. Transmission electron microscopy (TEM) image (A: $50,000 \times)$ and energy dispersive spectroscopy (EDS) analysis (B) of nanosized $\mathrm{Nd}_{2} \mathrm{O}_{3}$ collected from the high dose chamber. Aciniform aggregates and agglomerates were remarkably shown. Bar $=0.1 \mu \mathrm{m}$.

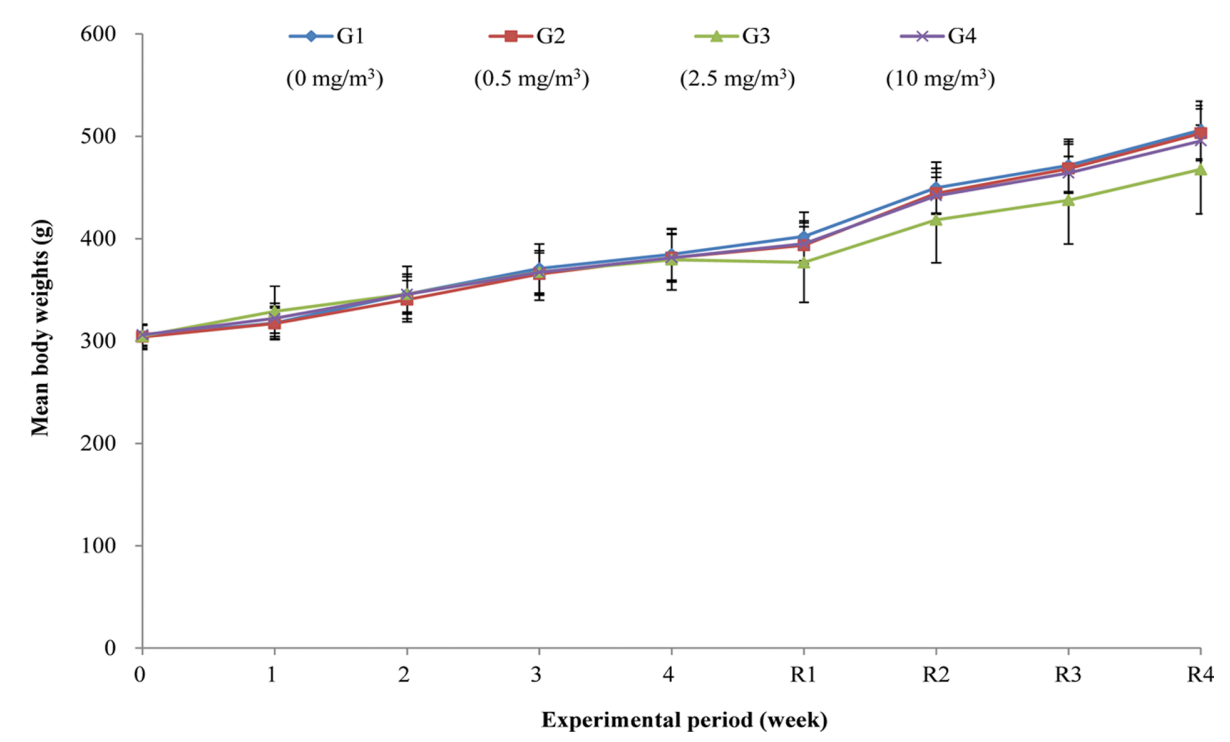

Fig. 3. Mean body weight of rats exposed to $0(\diamond), 0.5(\boldsymbol{\square}), 2.5(\boldsymbol{\Delta})$, and $10(\times) \mathrm{mg} / \mathrm{m}^{3}$ nano-sized $\mathrm{Nd}_{2} \mathrm{O}_{3}$ during the experimental period. Values are expressed as means $\pm \mathrm{SD}$ [exposure period; $n=15(\mathrm{G} 3)$ or $16(\mathrm{G} 1, \mathrm{G} 2, \mathrm{G} 4)$, recovery period; $n=7(\mathrm{G} 3)$ or $8(\mathrm{G} 1, \mathrm{G} 2, \mathrm{G} 4)$ ]. 
Table 4. Hematological values of rats after 28-day exposure of nano-sized $\mathrm{Nd}_{2} \mathrm{O}_{3}$

\begin{tabular}{lcccc}
\hline \hline \multirow{2}{*}{ Parameter } & \multicolumn{3}{c}{ Dose $\left(\mathrm{mg} / \mathrm{m}^{3}\right)$} \\
\cline { 2 - 5 } & 0 & 0.5 & 2.5 & 10 \\
\hline No. of animals examined & 8 & 8 & 8 & 8 \\
RBC $\left(10^{6} / \mu \mathrm{L}\right)$ & $8.40 \pm 0.41^{\mathrm{a}}$ & $8.69 \pm 0.30$ & $8.82 \pm 0.60$ & $8.58 \pm 0.51$ \\
Hemoglobin $(\mathrm{g} / \mathrm{dL})$ & $15.1 \pm 0.71$ & $15.7 \pm 0.48$ & $15.8 \pm 0.65$ & $15.4 \pm 0.63$ \\
Hematocrit $(\%)$ & $42.3 \pm 1.63$ & $43.8 \pm 1.49$ & $44.2 \pm 1.97$ & $42.7 \pm 1.80$ \\
MCV $(\mathrm{fL})$ & $50.4 \pm 1.04$ & $50.4 \pm 1.63$ & $50.2 \pm 1.50$ & $49.8 \pm 1.37$ \\
MCH $(\mathrm{pg})$ & $18.0 \pm 0.55$ & $18.1 \pm 0.60$ & $17.9 \pm 0.56$ & $17.9 \pm 0.70$ \\
MCHC $(\mathrm{g} / \mathrm{dL})$ & $35.7 \pm 0.51$ & $35.8 \pm 0.25$ & $35.6 \pm 0.35$ & $36.5 \pm 0.96$ \\
RDW $(\%)$ & $12.4 \pm 0.76$ & $12.1 \pm 0.46$ & $11.9 \pm 0.68$ & $12.9 \pm 2.04$ \\
Platelet $\left(10^{3} / \mu \mathrm{L}\right)$ & $925 \pm 121$ & $987 \pm 113$ & $772 \pm 157$ & $984 \pm 51.4$ \\
MPV $(\mathrm{fL})$ & $9.16 \pm 0.66$ & $8.91 \pm 0.49$ & $8.85 \pm 0.74$ & $9.76 \pm 1.68$ \\
WBC $\left(10^{3} / \mu \mathrm{L}\right)$ & $3.90 \pm 1.20$ & $4.82 \pm 0.85$ & $4.49 \pm 2.31$ & $4.08 \pm 1.65$ \\
Neutrophils $\left(10^{3} / \mu \mathrm{L}\right)$ & $0.79 \pm 0.29$ & $0.99 \pm 0.25$ & $1.08 \pm 0.61$ & $0.78 \pm 0.21$ \\
Lymphocytes $\left(10^{3} / \mu \mathrm{L}\right)$ & $2.97 \pm 1.01$ & $3.65 \pm 0.70$ & $3.22 \pm 1.68$ & $3.12 \pm 1.42$ \\
Monocytes $\left(10^{3} / \mu \mathrm{L}\right)$ & $0.06 \pm 0.03$ & $0.08 \pm 0.03$ & $0.10 \pm 0.07$ & $0.09 \pm 0.04$ \\
Eosinophils $\left(10^{3} / \mu \mathrm{L}\right)$ & $0.05 \pm 0.04$ & $0.06 \pm 0.02$ & $0.06 \pm 0.02$ & $0.05 \pm 0.03$ \\
Basophils $\left(10^{3} / \mu \mathrm{L}\right)$ & $0.01 \pm 0.01$ & $0.01 \pm 0.01$ & $0.01 \pm 0.01$ & $0.01 \pm 0.01$ \\
\hline
\end{tabular}

$\mathrm{RBC}$, red blood cell count; $\mathrm{MCV}$, mean corpuscular volume; $\mathrm{MCH}$, mean corpuscular hemoglobin; $\mathrm{MCHC}$, mean corpuscular hemoglobin concentration; RDW, red cell distribution width; MPV, mean platelet volume; WBC, white blood cell count.

${ }^{\mathrm{a}}$ Values are expressed as means \pm SD.

Table 5. Hematological values of rats after 28-day recovery

\begin{tabular}{lcccc}
\hline \hline & \multicolumn{3}{c}{ Dose $\left(\mathrm{mg} / \mathrm{m}^{3}\right)$} \\
\cline { 2 - 5 } Parameter & 0 & 0.5 & 2.5 & 10 \\
\hline No. of animals examined & 8 & 8 & 7 & 8 \\
RBC $\left(10^{6} / \mu \mathrm{L}\right)$ & $8.63 \pm 0.32^{\mathrm{a}}$ & $8.71 \pm 0.27$ & $9.20 \pm 0.46$ & $9.09 \pm 0.37$ \\
Hemoglobin $(\mathrm{g} / \mathrm{dL})$ & $15.6 \pm 0.43$ & $15.7 \pm 0.86$ & $15.9 \pm 0.53$ & $16.0 \pm 0.53$ \\
Hematocrit $(\%)$ & $44.9 \pm 1.49$ & $45.0 \pm 2.08$ & $46.9 \pm 2.19$ & $46.4 \pm 1.76$ \\
MCV $(\mathrm{fL})$ & $52.0 \pm 0.83$ & $51.6 \pm 1.73$ & $51.0 \pm 1.25$ & $51.1 \pm 0.69$ \\
MCH $(\mathrm{pg})$ & $18.1 \pm 0.55$ & $18.0 \pm 0.70$ & $17.3 \pm 0.69$ & $17.6 \pm 0.39$ \\
MCHC $(\mathrm{g} / \mathrm{dL})$ & $34.7 \pm 0.78$ & $34.8 \pm 0.61$ & $33.9 \pm 0.99$ & $34.5 \pm 0.59$ \\
RDW $(\%)$ & $13.9 \pm 0.56$ & $14.1 \pm 0.27$ & $13.4 \pm 0.39$ & $14.6 \pm 2.26$ \\
Platelet $\left(10^{3} / \mu \mathrm{L}\right)$ & $983 \pm 51.0$ & $971 \pm 61.2$ & $944 \pm 111$ & $993 \pm 94.2$ \\
MPV $(\mathrm{fL})$ & $7.79 \pm 0.34$ & $7.68 \pm 0.33$ & $7.53 \pm 0.27$ & $7.83 \pm 0.67$ \\
WBC $\left(10^{3} / \mu \mathrm{L}\right)$ & $5.23 \pm 0.75$ & $5.37 \pm 1.14$ & $5.43 \pm 0.58$ & $5.76 \pm 1.25$ \\
Neutrophils $\left(10^{3} / \mu \mathrm{L}\right)$ & $1.01 \pm 0.14$ & $1.23 \pm 0.44$ & $1.34 \pm 0.26$ & $1.28 \pm 0.23$ \\
Lymphocytes $\left(10^{3} / \mu \mathrm{L}\right)$ & $3.94 \pm 0.70$ & $3.90 \pm 0.93$ & $3.84 \pm 0.35$ & $4.23 \pm 1.13$ \\
Monocytes $\left(10^{3} / \mu \mathrm{L}\right)$ & $0.13 \pm 0.02$ & $0.11 \pm 0.06$ & $0.12 \pm 0.04$ & $0.11 \pm 0.03$ \\
Eosinophils $\left(10^{3} / \mu \mathrm{L}\right)$ & $0.08 \pm 0.02$ & $0.07 \pm 0.04$ & $0.07 \pm 0.02$ & $0.07 \pm 0.03$ \\
Basophils $\left(10^{3} / \mu \mathrm{L}\right)$ & $0.01 \pm 0.00$ & $0.01 \pm 0.01$ & $0.01 \pm 0.00$ & $0.01 \pm 0.01$ \\
\hline
\end{tabular}

$\mathrm{RBC}$, red blood cell count; $\mathrm{MCV}$, mean corpuscular volume; $\mathrm{MCH}$, mean corpuscular hemoglobin; $\mathrm{MCHC}$, mean corpuscular hemoglobin concentration; RDW, red cell distribution width; MPV, mean platelet volume; WBC, white blood cell count.

${ }^{a}$ Values are expressed as means \pm SD.

the exposure and recovery periods (data not shown). Moreover, no significant differences in body weight were observed between the treatment groups and controls (Fig. 3).

Hematology and serum biochemistry. The results of hematology and serum biochemistry analyses are presented in Table 4, 5, 6, and 7. There were no significant differences between the treatment groups and controls.
Necropsy and organ weight. There were no treatmentrelated gross findings in any of the treated groups (data not shown).

The results of the absolute and relative organ weights are presented in Table 8 . The absolute and relative lung weights increased in all treatment groups with a statistically significant difference when compared with those in the control group, and a clear dose-dependent relationship was observed 
Table 6. Serum biochemical values of rats after 28-day exposure of nano-sized $\mathrm{Nd}_{2} \mathrm{O}_{3}$

\begin{tabular}{lcccc}
\hline \hline & \multicolumn{3}{c}{ Dose $\left(\mathrm{mg} / \mathrm{m}^{3}\right)$} \\
\cline { 2 - 5 } Parameter & 0 & 0.5 & 2.5 & 10 \\
\hline No. of animals examined & 8 & 8 & 8 & 8 \\
AST (IU/L) & $94.4 \pm 20.1^{\mathrm{a}}$ & $92.2 \pm 18.8$ & $105 \pm 31.5$ & $110 \pm 22.0$ \\
ALT (IU/L) & $56.1 \pm 7.30$ & $59.7 \pm 11.9$ & $58.0 \pm 11.0$ & $54.2 \pm 8.45$ \\
ALP (IU/L) & $598 \pm 51.5$ & $677 \pm 101$ & $667 \pm 100$ & $607 \pm 138$ \\
GGT (IU/L) & $0.69 \pm 0.60$ & $0.38 \pm 0.24$ & $0.28 \pm 0.32$ & $0.45 \pm 0.62$ \\
CPK (IU/L) & $436 \pm 225$ & $290 \pm 115$ & $334 \pm 209$ & $414 \pm 131$ \\
LDH (IU/L) & $1331 \pm 622$ & $842 \pm 309$ & $1030 \pm 724$ & $1404 \pm 385$ \\
TBil (mg/dL) & $0.17 \pm 0.02$ & $0.18 \pm 0.03$ & $0.21 \pm 0.04$ & $0.19 \pm 0.03$ \\
Glucose (mg/dL) & $124 \pm 11.1$ & $127 \pm 12.6$ & $120 \pm 15.6$ & $111 \pm 8.52$ \\
TCho (mg/dL) & $59.3 \pm 10.2$ & $58.5 \pm 9.14$ & $54.8 \pm 7.43$ & $54.4 \pm 12.0$ \\
Triglyceride (mg/dL) & $38.7 \pm 11.6$ & $37.6 \pm 13.7$ & $34.5 \pm 11.1$ & $26.2 \pm 6.10$ \\
TP (g/dL) & $6.23 \pm 0.13$ & $6.34 \pm 0.12$ & $6.35 \pm 0.16$ & $6.30 \pm 0.23$ \\
Albumin (g/dL) & $4.21 \pm 0.08$ & $4.29 \pm 0.10$ & $4.28 \pm 0.10$ & $4.23 \pm 0.15$ \\
BUN (mg/dL) & $16.6 \pm 1.74$ & $18.5 \pm 1.18$ & $17.7 \pm 1.98$ & $14.8 \pm 1.00$ \\
Creatinine (mg/dL) & $0.42 \pm 0.02$ & $0.42 \pm 0.02$ & $0.44 \pm 0.02$ & $0.43 \pm 0.02$ \\
IP (mg/dL) & $7.43 \pm 0.49$ & $7.70 \pm 0.37$ & $7.73 \pm 0.35$ & $7.84 \pm 0.44$ \\
Ca ${ }^{++}$(mg/dL) & $10.2 \pm 0.21$ & $10.1 \pm 0.16$ & $10.1 \pm 0.21$ & $10.1 \pm 0.21$ \\
\hline AST, aspartate amingtransferyyyy
\end{tabular}

AST, aspartate aminotransferase; ALT, alanine aminotransferase; ALP, alkaline phosphatase; GGT, gamma glutamyl transferase; CPK, creatine phosphokinase; LDH, lactate dehydrogenase; TBil, total bilirubin; TCho, total cholesterol; TP, total protein; BUN, blood urea nitrogen; IP, inorganic phosphorus; Ca, calcium.

${ }^{a}$ Values are expressed as means $\pm \mathrm{SD}$.

Table 7. Serum biochemical values of rats after 28-day recovery

\begin{tabular}{|c|c|c|c|c|}
\hline \multirow{2}{*}{ Parameter } & \multicolumn{4}{|c|}{ Dose $\left(\mathrm{mg} / \mathrm{m}^{3}\right)$} \\
\hline & 0 & 0.5 & 2.5 & 10 \\
\hline No. of animals examined & 8 & 8 & 7 & 8 \\
\hline $\operatorname{AST}(\mathrm{IU} / \mathrm{L})$ & $85.5 \pm 9.79^{\mathrm{a}}$ & $83.8 \pm 11.0$ & $84.6 \pm 11.5$ & $92.3 \pm 19.8$ \\
\hline ALT (IU/L) & $54.1 \pm 6.10$ & $62.4 \pm 15.0$ & $63.3 \pm 10.3$ & $59.4 \pm 9.73$ \\
\hline ALP (IU/L) & $618 \pm 140$ & $617 \pm 176$ & $649 \pm 189$ & $604 \pm 101$ \\
\hline GGT (IU/L) & $0.99 \pm 0.71$ & $1.06 \pm 0.49$ & $1.21 \pm 0.63$ & $0.95 \pm 0.45$ \\
\hline CPK (IU/L) & $262 \pm 134$ & $274 \pm 196$ & $217 \pm 135$ & $203 \pm 124$ \\
\hline LDH (IU/L) & $910 \pm 529$ & $984 \pm 796$ & $718 \pm 542$ & $781 \pm 568$ \\
\hline TBil (mg/dL) & $0.10 \pm 0.04$ & $0.10 \pm 0.04$ & $0.10 \pm 0.05$ & $0.09 \pm 0.03$ \\
\hline Glucose (mg/dL) & $135 \pm 15.8$ & $125 \pm 12.5$ & $139 \pm 17.7$ & $130 \pm 9.64$ \\
\hline TCho (mg/dL) & $78.7 \pm 16.9$ & $75.1 \pm 12.3$ & $73.5 \pm 12.1$ & $64.1 \pm 8.41$ \\
\hline Triglyceride (mg/dL) & $48.2 \pm 14.5$ & $47.6 \pm 12.7$ & $40.4 \pm 12.9$ & $42.7 \pm 11.9$ \\
\hline $\mathrm{TP}(\mathrm{g} / \mathrm{dL})$ & $7.26 \pm 0.26$ & $7.08 \pm 0.30$ & $7.31 \pm 0.34$ & $7.19 \pm 0.25$ \\
\hline Albumin $(\mathrm{g} / \mathrm{dL})$ & $4.75 \pm 0.14$ & $4.69 \pm 0.18$ & $4.76 \pm 0.16$ & $4.71 \pm 0.15$ \\
\hline BUN (mg/dL) & $21.2 \pm 2.70$ & $21.5 \pm 3.92$ & $20.4 \pm 2.34$ & $22.2 \pm 3.90$ \\
\hline Creatinine (mg/dL) & $0.51 \pm 0.05$ & $0.49 \pm 0.04$ & $0.51 \pm 0.02$ & $0.52 \pm 0.02$ \\
\hline IP $(\mathrm{mg} / \mathrm{dL})$ & $8.08 \pm 0.93$ & $8.11 \pm 0.62$ & $8.49 \pm 0.83$ & $7.95 \pm 0.74$ \\
\hline $\mathrm{Ca}^{++}(\mathrm{mg} / \mathrm{dL})$ & $11.8 \pm 0.66$ & $11.2 \pm 0.44$ & $11.3 \pm 0.44$ & $11.0 \pm 0.41$ \\
\hline
\end{tabular}

AST, aspartate aminotransferase; ALT, alanine aminotransferase; ALP, alkaline phosphatase; GGT, gamma glutamyl transferase; CPK, creatine phosphokinase; LDH, lactate dehydrogenase; TBil, total bilirubin; TCho, total cholesterol; TP, total protein; BUN, blood urea nitrogen; IP, inorganic phosphorus; Ca, calcium.

alues are expressed as means \pm SD.

at the end of the 28-day recovery period. The absolute lung weight increased by 54,69 , and $61 \%$, respectively, in the $0.5,2.5$, and $10 \mathrm{mg} / \mathrm{m}^{3}$ treatment groups, and increased by 24,80 , and $105 \%$, respectively, in the recovery groups, when compared with those in the control group.
Histopathology. The results of the histopathology examinations are presented in Table 9, Fig. 4, and 5. Alveolar proteinosis of the lung was observed in all cases in the $0.5 \mathrm{mg} / \mathrm{m}^{3}$ and higher dose treatment groups, and in six, seven, and eight cases, respectively, in the $0.5,2.5$, and 10 
Table 8. Absolute and relative organ weights of rats treated with nano-sized $\mathrm{Nd}_{2} \mathrm{O}_{3}$ for 28 days

\begin{tabular}{|c|c|c|c|c|}
\hline \multirow{2}{*}{ Parameter (after exposure) } & \multicolumn{4}{|c|}{ Dose $\left(\mathrm{mg} / \mathrm{m}^{3}\right)$} \\
\hline & 0 & 0.5 & 2.5 & 10 \\
\hline No. of animals examined & 8 & 8 & 8 & 8 \\
\hline Body weight (g) & $346.3 \pm 18.53^{\mathrm{a}}$ & $345.4 \pm 21.98$ & $359.2 \pm 19.99$ & $347.1 \pm 19.53$ \\
\hline Lung (g) & $1.391 \pm 0.094$ & $2.141 \pm 0.270^{* *}$ & $2.345 \pm 0.114^{* *}$ & $2.238 \pm 0.149^{* *}$ \\
\hline Per body weight $(\%)$ & $0.402 \pm 0.016$ & $0.619 \pm 0.059^{* *}$ & $0.655 \pm 0.047^{* *}$ & $0.645 \pm 0.034^{* *}$ \\
\hline Liver $(\mathrm{g})$ & $9.395 \pm 0.664$ & $9.490 \pm 0.867$ & $9.843 \pm 0.981$ & $9.189 \pm 0.585$ \\
\hline Per body weight (\%) & $2.713 \pm 0.110$ & $2.745 \pm 0.124$ & $2.735 \pm 0.155$ & $2.650 \pm 0.149$ \\
\hline Kidney (g) & $2.325 \pm 0.161$ & $2.424 \pm 0.142$ & $2.456 \pm 0.218$ & $2.345 \pm 0.118$ \\
\hline Per body weight $(\%)$ & $0.672 \pm 0.043$ & $0.703 \pm 0.043$ & $0.683 \pm 0.040$ & $0.676 \pm 0.032$ \\
\hline Spleen $(\mathrm{g})$ & $0.651 \pm 0.085$ & $0.644 \pm 0.060$ & $0.657 \pm 0.070$ & $0.610 \pm 0.072$ \\
\hline Per body weight $(\%)$ & $0.188 \pm 0.018$ & $0.186 \pm 0.011$ & $0.183 \pm 0.014$ & $0.176 \pm 0.021$ \\
\hline Brain $(g)$ & $2.038 \pm 0.084$ & $2.006 \pm 0.049$ & $2.044 \pm 0.094$ & $1.981 \pm 0.086$ \\
\hline Per body weight $(\%)$ & $0.590 \pm 0.033$ & $0.583 \pm 0.038$ & $0.571 \pm 0.041$ & $0.572 \pm 0.035$ \\
\hline \multirow{2}{*}{ Parameter (after recovery) } & \multicolumn{4}{|c|}{ Dose $\left(\mathrm{mg} / \mathrm{m}^{3}\right)$} \\
\hline & 0 & 0.5 & 2.5 & 10 \\
\hline No. of animals examined & 8 & 8 & 7 & 8 \\
\hline Body weight (g) & $499.9 \pm 28.93$ & $495.9 \pm 25.25$ & $464.6 \pm 44.10$ & $488.6 \pm 30.07$ \\
\hline Lung (g) & $1.709 \pm 0.132$ & $2.118 \pm 0.191^{* *}$ & $3.077 \pm 0.482^{* *}$ & $3.495 \pm 0.460^{* *}$ \\
\hline Per body weight (\%) & $0.342 \pm 0.027$ & $0.427 \pm 0.030^{* *}$ & $0.661 \pm 0.063^{* *}$ & $0.716 \pm 0.082^{* *}$ \\
\hline Liver $(g)$ & $13.75 \pm 1.675$ & $13.49 \pm 0.918$ & $12.39 \pm 1.509$ & $13.02 \pm 1.021$ \\
\hline Per body weight (\%) & $2.745 \pm 0.224$ & $2.720 \pm 0.144$ & $2.664 \pm 0.135$ & $2.667 \pm 0.151$ \\
\hline Kidney $(\mathrm{g})$ & $3.071 \pm 0.292$ & $2.974 \pm 0.173$ & $2.829 \pm 0.357$ & $2.890 \pm 0.148$ \\
\hline Per body weight $(\%)$ & $0.614 \pm 0.033$ & $0.600 \pm 0.033$ & $0.610 \pm 0.058$ & $0.592 \pm 0.029$ \\
\hline Spleen $(\mathrm{g})$ & $0.926 \pm 0.095$ & $0.862 \pm 0.116$ & $0.828 \pm 0.080$ & $0.853 \pm 0.034$ \\
\hline Per body weight $(\%)$ & $0.185 \pm 0.017$ & $0.174 \pm 0.021$ & $0.178 \pm 0.010$ & $0.175 \pm 0.013$ \\
\hline Brain $(g)$ & $2.140 \pm 0.098$ & $2.113 \pm 0.055$ & $2.073 \pm 0.072$ & $2.066 \pm 0.099$ \\
\hline Per body weight $(\%)$ & $0.428 \pm 0.014$ & $0.427 \pm 0.018$ & $0.450 \pm 0.049$ & $0.425 \pm 0.041$ \\
\hline
\end{tabular}

Values are expressed as means \pm SD.

** Significantly different from vehicle control at $p<0.01$.

$\mathrm{mg} / \mathrm{m}^{3}$ recovery groups. Hyperplasia/hypertrophy of alveolar type II cells in the lung was observed in one case each in the 0.5 and $10 \mathrm{mg} / \mathrm{m}^{3}$ treatment groups and in two cases in the $10 \mathrm{mg} / \mathrm{m}^{3}$ recovery group. Aggregation of alveolar macrophages in the lung was observed in five and one cases, respectively, in the 0.5 and $10 \mathrm{mg} / \mathrm{m}^{3}$ treatment groups and in six, five, and two cases, respectively, in the $0.5,2.5$, and $10 \mathrm{mg} / \mathrm{m}^{3}$ recovery groups. Alveolar/perivascular infiltration of inflammatory cells in the lung was observed in two, three, five, and four cases, respectively, in the control, 0.5 , 2.5 , and $10 \mathrm{mg} / \mathrm{m}^{3}$ treatment groups and in three, one, three, and four cases, respectively, in the control, 0.5, 2.5, and 10 $\mathrm{mg} / \mathrm{m}^{3}$ recovery groups. Aggregation of macrophages in the hilar lymph node was observed in one and two cases, respectively, in the 0.5 and $10 \mathrm{mg} / \mathrm{m}^{3}$ treatment groups and in two and four cases, respectively, in the 2.5 and $10 \mathrm{mg} / \mathrm{m}^{3}$ recovery groups. The severity of alveolar proteinosis and infiltration of inflammatory cells in the lung, and aggregation of macrophages in the hilar lymph node, increased in a dose-dependent manner in the recovery groups. No treatment-related lesions were observed in other organs except for the lung and hilar lymph node.
Analysis of BALF. The BALF pellets obtained from all treatment groups except for those in the $0.5 \mathrm{mg} / \mathrm{m}^{3}$ recovery group had an opaque, milky appearance. The results of BALF cellular analysis are presented in Table 10. The number of total cells, macrophages, and neutrophils increased in all treatment groups. While these changes generally decreased in the 0.5 and $2.5 \mathrm{mg} / \mathrm{m}^{3}$ recovery groups, increases were observed in the $10 \mathrm{mg} / \mathrm{m}^{3}$ recovery group, showing a clear dose dependency. A statistically significant difference in the results of BALF analysis was observed for all treatment groups, except for the macrophage count in the $0.5 \mathrm{mg} / \mathrm{m}^{3}$ treatment group, total cell and macrophage counts in the 0.5 and $2.5 \mathrm{mg} / \mathrm{m}^{3}$ recovery groups, and neutrophil count in the $0.5 \mathrm{mg} / \mathrm{m}^{3}$ recovery group, when compared with those in the control group. There were no significant changes in lymphocyte and eosinophil counts (data not shown). Foamy, distorted, or collapsed alveolar macrophages were observed in BALF cells of all treatment groups; however, BALF cells of the $0.5 \mathrm{mg} / \mathrm{m}^{3}$ recovery group were very similar in appearance to those from the control group (Fig. 6, 7).

The results of the biochemical analysis for the BALF supernatant are presented in Table 10. LDH, albumin, IL-6, and 
Table 9. Histopathologic findings of rats treated with nano-sized $\mathrm{Nd}_{2} \mathrm{O}_{3}$ for 28 days

\begin{tabular}{|c|c|c|c|c|c|}
\hline \multirow{2}{*}{ Organ/Findings (after exposure) } & \multirow{2}{*}{ Grade } & \multicolumn{4}{|c|}{ Dose $\left(\mathrm{mg} / \mathrm{m}^{3}\right)$} \\
\hline & & 0 & 0.5 & 2.5 & 10 \\
\hline No. of animals examined & & 8 & 8 & 8 & 8 \\
\hline Normal appearance & & 6 & 0 & 0 & 0 \\
\hline \multicolumn{6}{|l|}{ Lung } \\
\hline \multirow[t]{3}{*}{ Alveolar proteinosis } & \pm & 0 & 7 & 7 & 6 \\
\hline & + & 0 & 1 & 1 & 1 \\
\hline & ++ & 0 & 0 & 0 & 1 \\
\hline Hyperplasia/hypertrophy, alveolar type II cells & \pm & 0 & 1 & 0 & 1 \\
\hline \multirow[t]{2}{*}{ Aggregation, alveolar macrophages } & \pm & 0 & 2 & 0 & 1 \\
\hline & + & 0 & 3 & 0 & 0 \\
\hline \multirow[t]{3}{*}{ Infiltration, inflammatory cells, alveolar/perivascular } & \pm & 2 & 3 & 5 & 2 \\
\hline & + & 0 & 0 & 0 & 1 \\
\hline & ++ & 0 & 0 & 0 & 1 \\
\hline \multicolumn{6}{|l|}{ Lymph node, hilar } \\
\hline \multirow[t]{2}{*}{ Aggregation, macrophages } & \pm & 0 & 1 & 0 & 1 \\
\hline & + & 0 & 0 & 0 & 1 \\
\hline \multirow{2}{*}{ Organ/Findings (after recovery) } & \multirow{2}{*}{ Grade } & \multicolumn{4}{|c|}{ Dose $\left(\mathrm{mg} / \mathrm{m}^{3}\right)$} \\
\hline & & 0 & 0.5 & 2.5 & 10 \\
\hline No. of animals examined & & 8 & 8 & 7 & 8 \\
\hline Normal appearance & & 5 & 1 & 0 & 0 \\
\hline \multicolumn{6}{|l|}{ Lung } \\
\hline \multirow[t]{4}{*}{ Alveolar proteinosis } & \pm & 0 & 6 & 5 & 0 \\
\hline & + & 0 & 0 & 1 & 6 \\
\hline & ++ & 0 & 0 & 0 & 2 \\
\hline & +++ & 0 & 0 & 1 & 0 \\
\hline \multirow[t]{2}{*}{ Hyperplasia/hypertrophy, alveolar type II cells } & \pm & 0 & 0 & 0 & 1 \\
\hline & + & 0 & 0 & 0 & 1 \\
\hline \multirow[t]{3}{*}{ Aggregation, alveolar macrophages } & \pm & 0 & 6 & 3 & 1 \\
\hline & + & 0 & 0 & 1 & 1 \\
\hline & ++ & 0 & 0 & 1 & 0 \\
\hline \multirow[t]{3}{*}{ Infiltration, inflammatory cells, alveolar/perivascular } & \pm & 3 & 1 & 2 & 2 \\
\hline & + & 0 & 0 & 1 & 1 \\
\hline & +++ & 0 & 0 & 0 & 1 \\
\hline \multicolumn{6}{|l|}{ Lymph node, hilar } \\
\hline \multirow[t]{4}{*}{ Aggregation, macrophages } & \pm & 0 & 0 & 1 & 0 \\
\hline & + & 0 & 0 & 1 & 0 \\
\hline & ++ & 0 & 0 & 0 & 3 \\
\hline & +++ & 0 & 0 & 0 & 1 \\
\hline
\end{tabular}

Grade: \pm , minimal; +, mild; ++, moderate; and +++, marked.

TNF- $\alpha$ increased in all treatment groups. While this change was generally less apparent in the 0.5 and $2.5 \mathrm{mg} / \mathrm{m}^{3}$ recovery groups, it increased, except for the change in IL- 6 , in the $10 \mathrm{mg} / \mathrm{m}^{3}$ recovery group, showing a clear dose-dependent relationship. A statistically significant difference was observed in results for all groups, except for the IL- 6 in the $0.5 \mathrm{mg}$ / $\mathrm{m}^{3}$ treatment group, albumin and TNF- $\alpha$ in the $0.5 \mathrm{mg} / \mathrm{m}^{3}$ recovery group, and IL- 6 in the 0.5 and $2.5 \mathrm{mg} / \mathrm{m}^{3}$ recovery groups, when compared with those in the control group.

Oxidative stress analysis. The results of oxidative stress analysis of the lung homogenate are presented in Table 11.
GSH content decreased significantly in the $2.5 \mathrm{mg} / \mathrm{m}^{3}$ and higher dose treatment groups in a dose-dependent manner, and also decreased in the $10 \mathrm{mg} / \mathrm{m}^{3}$ recovery group with a statistically significant difference. Although a statistically significant decrease was observed in catalase activity of the $0.5 \mathrm{mg} / \mathrm{m}^{3}$ treatment group, it was not dose-dependent.

Measurement of neodymium levels. The results of neodymium concentration measurements for major organs and blood are presented in Table 12. Neodymium levels increased in the major organs of all treatment groups in a dose-dependent manner. It accumulated as follows: lung $>>$ 

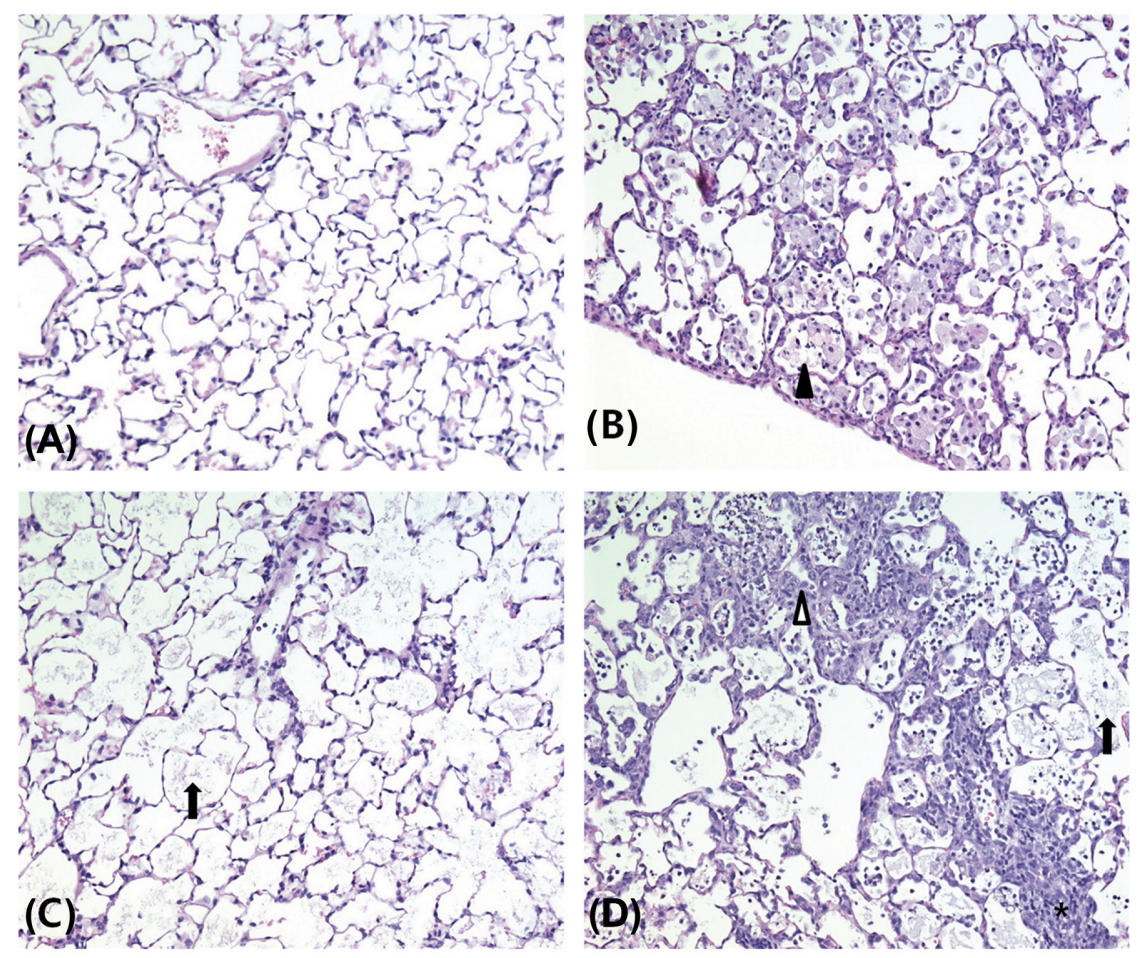

Fig. 4. Representative photographs of lung sections from the control $(A), 0.5(B), 2.5(C)$, and $10(D) \mathrm{mg} / \mathrm{m}^{3}$ treatment groups stained with hematoxylin and eosin $(200 \times)$. Alveolar proteinosis (arrow), alveolar macrophage aggregation (arrowhead), alveolar type II cells hyperplasia/hypertrophy (white arrowhead), and inflammatory cell infiltration (asterisk) were shown.
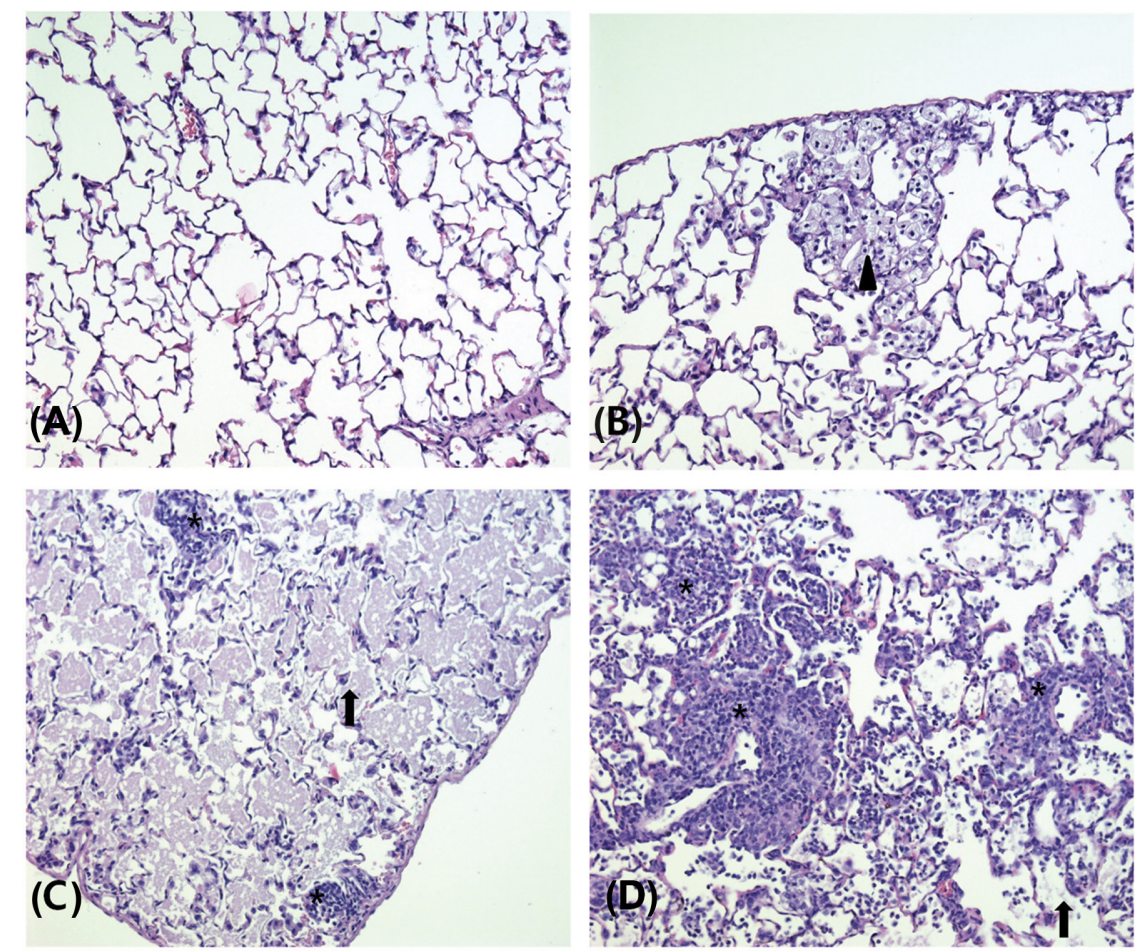

Fig. 5. Representative photographs of lung sections from the control (A), 0.5 (B), 2.5 (C), and 10 (D) $\mathrm{mg} / \mathrm{m}^{3}$ recovery groups stained with hematoxylin and eosin $(200 \times)$. Alveolar proteinosis (arrow), alveolar macrophage aggregation (arrowhead), and inflammatory cell infiltration (asterisk) were shown. 
Table 10. Analysis of bronchoalveolar lavage fluid from rats treated with nano-sized $\mathrm{Nd}_{2} \mathrm{O}_{3}$ for 28 days

\begin{tabular}{|c|c|c|c|c|}
\hline \multirow{2}{*}{$\begin{array}{l}\text { Parameter } \\
\text { (after 28-day exposure) }\end{array}$} & \multicolumn{4}{|c|}{ Dose $\left(\mathrm{mg} / \mathrm{m}^{3}\right)$} \\
\hline & 0 & 0.5 & 2.5 & 10 \\
\hline No. of animals examined & 8 & 8 & 8 & 8 \\
\hline Total cells $\left(10^{3} / \mu \mathrm{L}\right)$ & $0.78 \pm 0.31^{\mathrm{a}}$ & $29.3 \pm 16.0^{*}$ & $73.4 \pm 32.0^{* *}$ & $67.0 \pm 15.4^{* *}$ \\
\hline Macrophages $\left(10^{3} / \mu \mathrm{L}\right)$ & $0.77 \pm 0.30$ & $19.3 \pm 14.5$ & $46.8 \pm 28.7^{*}$ & $31.3 \pm 8.57^{* *}$ \\
\hline Neutrophils $\left(10^{3} / \mu \mathrm{L}\right)$ & $0.01 \pm 0.03$ & $9.96 \pm 3.10^{* * *}$ & $26.5 \pm 7.35^{* *}$ & $35.7 \pm 10.3^{* *}$ \\
\hline LDH (IU/L) & $89.9 \pm 33.4$ & $771 \pm 195^{* *}$ & $1117 \pm 179^{* * *}$ & $1080 \pm 345^{* *}$ \\
\hline Albumin (mg/dL) & $0.00 \pm 0.00$ & $18.3 \pm 7.18^{* *}$ & $33.9 \pm 13.3^{* *}$ & $36.9 \pm 19.1^{* *}$ \\
\hline IL-6 (pg/mL) & $71.6 \pm 9.63$ & $82.9 \pm 14.9$ & $102 \pm 8.90^{* *}$ & $114 \pm 22.2^{* * *}$ \\
\hline TNF- $\alpha(\mathrm{pg} / \mathrm{mL})$ & $3.64 \pm 0.41$ & $5.94 \pm 1.09^{*}$ & $7.89 \pm 2.49^{*}$ & $8.46 \pm 3.46^{*}$ \\
\hline \multirow{2}{*}{$\begin{array}{l}\text { Parameter } \\
\text { (after 28-day recovery) }\end{array}$} & \multicolumn{4}{|c|}{ Dose $\left(\mathrm{mg} / \mathrm{m}^{3}\right)$} \\
\hline & 0 & 0.5 & 2.5 & 10 \\
\hline No. of animals examined & 8 & 8 & 7 & 8 \\
\hline Total cells $\left(10^{3} / \mu \mathrm{L}\right)$ & $1.01 \pm 0.56$ & $9.91 \pm 10.3$ & $56.4 \pm 40.3$ & $96.9 \pm 43.1^{* * *}$ \\
\hline Macrophages $\left(10^{3} / \mu \mathrm{L}\right)$ & $0.98 \pm 0.55$ & $5.91 \pm 5.39$ & $32.5 \pm 28.7$ & $49.3 \pm 22.2^{* *}$ \\
\hline Neutrophils $\left(10^{3} / \mu \mathrm{L}\right)$ & $0.02 \pm 0.02$ & $3.99 \pm 5.02$ & $23.9 \pm 13.0^{*}$ & $47.3 \pm 21.1^{* *}$ \\
\hline LDH (IU/L) & $27.1 \pm 6.95$ & $109 \pm 57.9^{*}$ & $669 \pm 113^{* *}$ & $1216 \pm 264^{* *}$ \\
\hline Albumin (mg/dL) & $1.13 \pm 1.46$ & $4.38 \pm 4.66$ & $30.2 \pm 7.08^{* *}$ & $63.9 \pm 18.4^{* *}$ \\
\hline IL-6 (pg/mL) & $70.0 \pm 7.91$ & $72.8 \pm 10.3$ & $77.9 \pm 9.93$ & $100 \pm 16.5^{*}$ \\
\hline TNF- $\alpha(\mathrm{pg} / \mathrm{mL})$ & $2.63 \pm 0.69$ & $3.52 \pm 0.91$ & $7.59 \pm 2.10^{* *}$ & $9.34 \pm 3.47^{* *}$ \\
\hline
\end{tabular}

LDH, lactate dehydrogenase; IL-6, interleukin-6; TNF- $\alpha$, tumor necrosis factor-alpha.

aalues are expressed as means \pm SD.

${ }^{* * * *}$ Significantly different from vehicle control at $p<0.05$ and $p<0.01$.

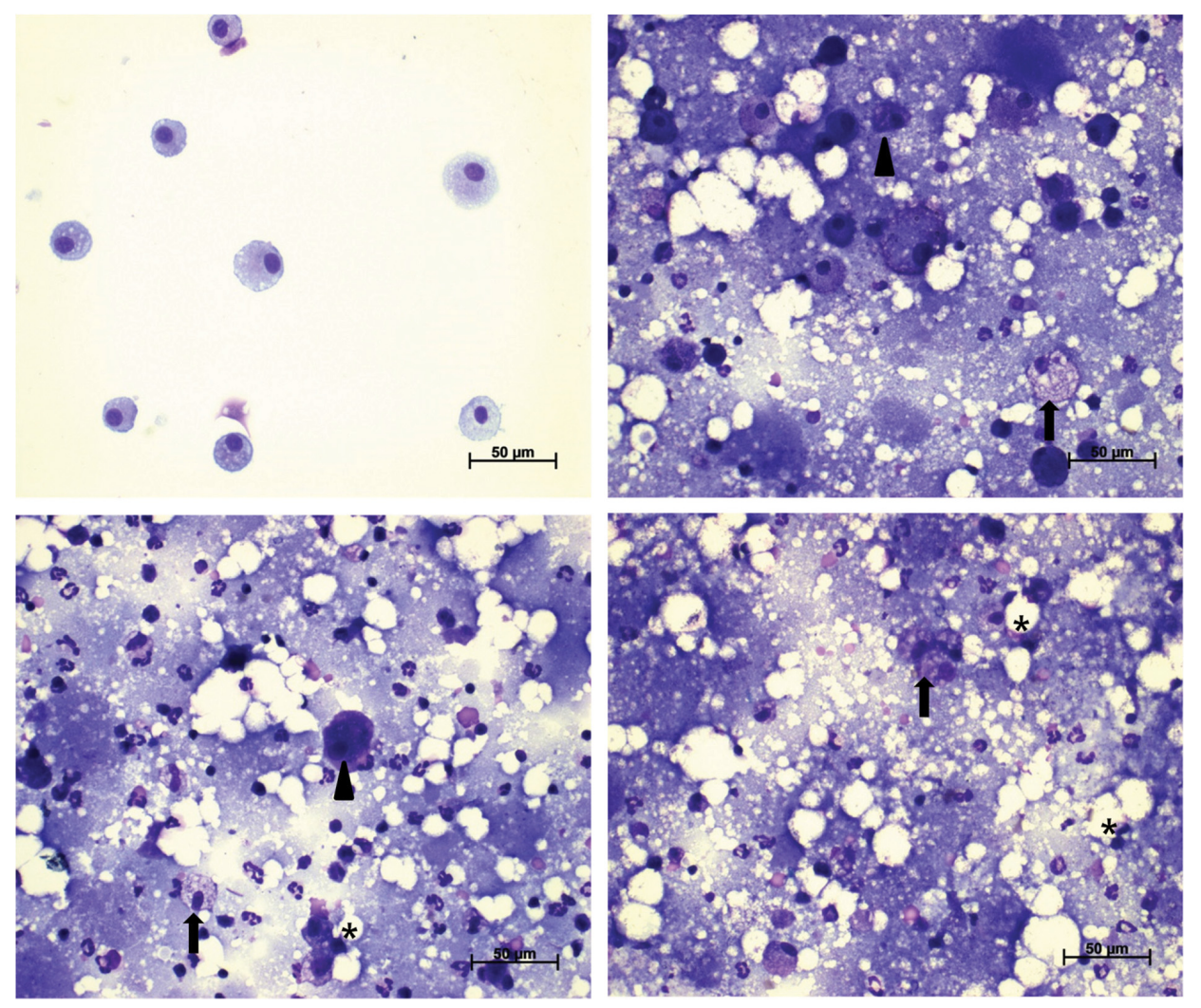

Fig. 6. Representative photographs of bronchoalveolar lavage fluids (BALF) from the control (A), 0.5 (B), 2.5 (C), and 10 (D) $\mathrm{mg} / \mathrm{m}^{3}$ treatment groups stained with a Diff-Quick staining solution $(400 \times)$. Morphological abnormalities (foamy; arrow, distorted; arrowhead, collapsed; asterisk) of alveolar macrophages were shown. 


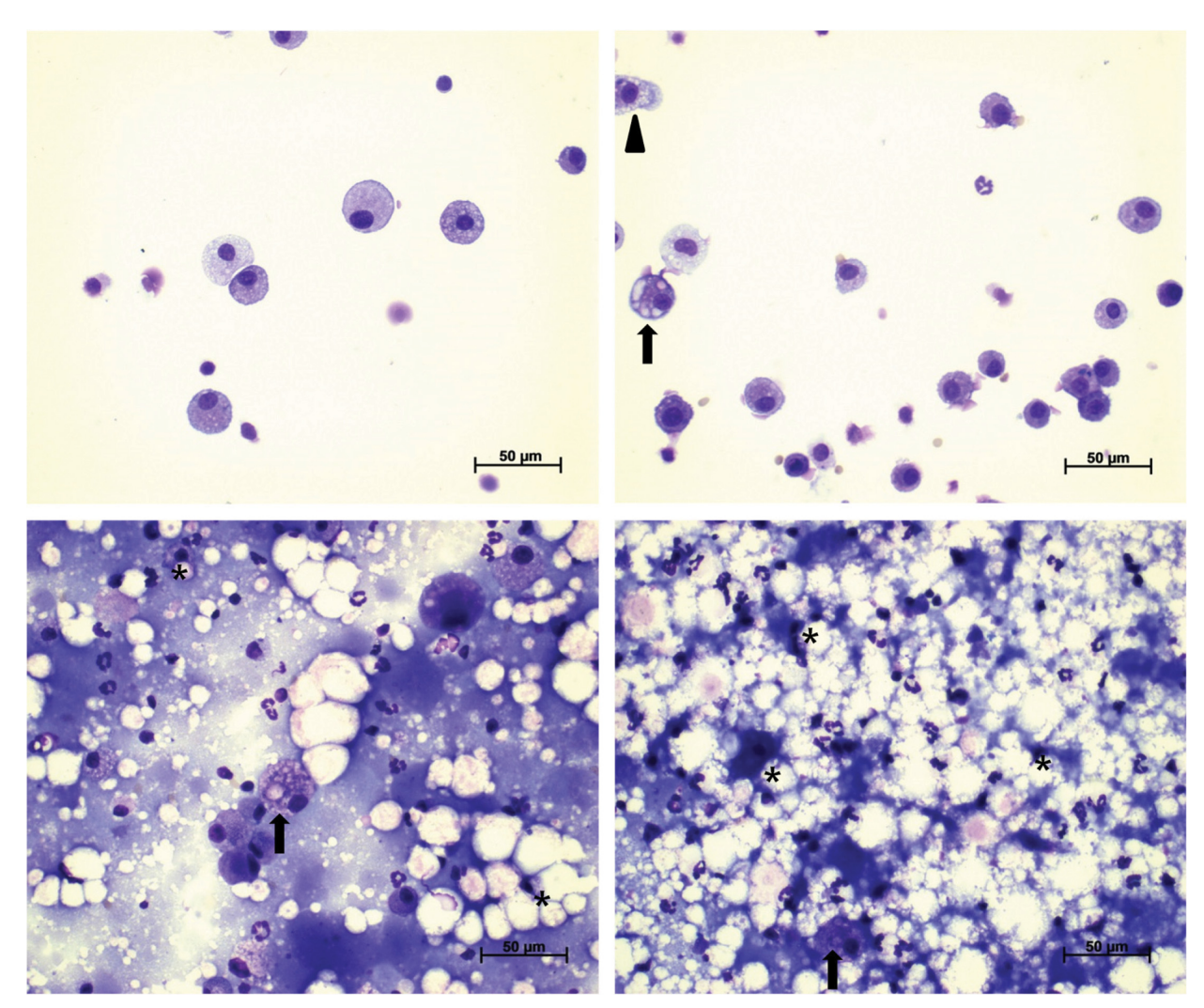

Fig. 7. Representative photographs of bronchoalveolar lavage fluids (BALF) from the control (A), 0.5 (B), 2.5 (C), and 10 (D) $\mathrm{mg} / \mathrm{m}^{3}$ recovery groups stained with a Diff-Quick staining solution $(400 \times)$. Morphological abnormalities (foamy; arrow, distorted; arrowhead, collapsed; asterisk) of alveolar macrophages were shown.

Table 11. Oxidative stress analysis of rat lung treated with nano-sized $\mathrm{Nd}_{2} \mathrm{O}_{3}$ for 28 days

\begin{tabular}{lcccc}
\hline \hline \multirow{2}{*}{$\begin{array}{l}\text { Parameter } \\
\text { (after 28-day exposure) }\end{array}$} & \multicolumn{4}{c}{ Dose $\left(\mathrm{mg} / \mathrm{m}^{3}\right)$} \\
\cline { 2 - 5 } & 0 & 0.5 & 2.5 & 8 \\
\hline No. of animals examined & 8 & 8 & 8 & $0.69 \pm 0.25^{* *}$ \\
GSH (nmol/mg protein) & $1.97 \pm 0.55^{\mathrm{a}}$ & $1.34 \pm 0.90$ & $0.48 \pm 0.22^{* *}$ \\
Catalase (units/mg protein) & $35.5 \pm 8.82$ & $22.3 \pm 2.37^{*}$ & $26.6 \pm 7.95$ & $38.3 \pm 4.59$ \\
\hline Parameter & \multicolumn{3}{c}{ Dose $\left(\mathrm{mg} / \mathrm{m}^{3}\right)$} \\
(after 28-day recovery) & 0 & 0.5 & 2.5 \\
\hline No. of animals examined & 8 & 8 & 7 \\
GSH (nmol/mg protein) & $1.49 \pm 0.59$ & $1.16 \pm 0.20$ & $1.05 \pm 0.19$ & $0.55 \pm 0.30^{*}$ \\
Catalase (units/mg protein) & $31.6 \pm 7.99$ & $28.9 \pm 4.03$ & $37.0 \pm 4.18$ & $33.0 \pm 6.83$ \\
\hline
\end{tabular}

\section{$\mathrm{GSH}$, glutathione.}

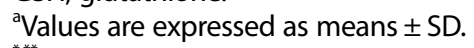

${ }^{* * * *}$ Significantly different from vehicle control at $p<0.05$ and $p<0.01$.

liver $>$ spleen $>$ brain $\fallingdotseq$ kidney, at the end of the 28-day exposure period, and the ratio of deposits in the lung to total organ deposits was approximately $97.4,99.4$, and $99.6 \%$, respectively, in the $0.5,2.5$, and $10 \mathrm{mg} / \mathrm{m}^{3}$ treatment groups. Neodymium levels decreased in the recovery groups and the rates of decrease in the lung were 21,34 , and $34 \%$ respectively, in the $0.5,2.5$ and $10 \mathrm{mg} / \mathrm{m}^{3}$ treatment groups. A statistically significant difference was observed in the neodymium levels of all organs, except for the spleen in the 0.5 and $2.5 \mathrm{mg} / \mathrm{m}^{3}$ treatment groups and the brain and spleen in the $0.5 \mathrm{mg} / \mathrm{m}^{3}$ and higher dose recovery groups, when compared with those in the control group. Neodymium levels were low in whole blood, with no clear dose-dependent relationship, although a statistically significant increase was observed in the $10 \mathrm{mg} / \mathrm{m}^{3}$ treatment group. 
Table 12. Tissue content of neodymium from rats treated with nano-sized $\mathrm{Nd}_{2} \mathrm{O}_{3}$ for 28 days

\begin{tabular}{|c|c|c|c|c|}
\hline \multirow{2}{*}{$\begin{array}{l}\text { Organ } \\
\text { (after 28-day exposure) }\end{array}$} & \multicolumn{4}{|c|}{ Dose $\left(\mathrm{mg} / \mathrm{m}^{3}\right)$} \\
\hline & 0 & 0.5 & 2.5 & 10 \\
\hline No. of animals examined & 8 & 8 & 8 & 8 \\
\hline Lung $(\mu \mathrm{g} / \mathrm{g})$ & $0.01 \pm 0.00^{\mathrm{a}}$ & $8.55 \pm 2.59^{* * *}$ & $59.0 \pm 19.2^{* *}$ & $183 \pm 25.9^{* *}$ \\
\hline Brain (ng/g) & $4.36 \pm 3.21$ & $25.6 \pm 6.95^{*}$ & $58.5 \pm 30.8^{*}$ & $108 \pm 55.3^{* *}$ \\
\hline Liver (ng/g) & $2.44 \pm 1.89$ & $121 \pm 34.2^{* * *}$ & $174 \pm 9.94^{* *}$ & $312 \pm 98.6^{* *}$ \\
\hline Spleen (ng/g) & $6.40 \pm 4.81$ & $34.1 \pm 21.3$ & $64.0 \pm 42.3$ & $177 \pm 108^{*}$ \\
\hline Kidney (ng/g) & $10.6 \pm 4.76$ & $43.9 \pm 12.3^{* * *}$ & $71.0 \pm 41.7^{*}$ & $91.9 \pm 40.8^{* *}$ \\
\hline Whole blood (ng/g) & $0.03 \pm 0.06$ & $0.04 \pm 0.05$ & $0.05 \pm 0.06$ & $0.15 \pm 0.07^{*}$ \\
\hline \multirow{2}{*}{$\begin{array}{l}\text { Organ } \\
\text { (after 28-day recovery) }\end{array}$} & \multicolumn{4}{|c|}{ Dose $\left(\mathrm{mg} / \mathrm{m}^{3}\right)$} \\
\hline & 0 & 0.5 & 2.5 & 10 \\
\hline No. of animals examined & 8 & 8 & 7 & 8 \\
\hline Lung $(\mu \mathrm{g} / \mathrm{g})$ & $0.01 \pm 0.01$ & $6.74 \pm 2.08^{* * *}$ & $38.7 \pm 12.5^{* * *}$ & $120 \pm 11.3^{* * *}$ \\
\hline Brain (ng/g) & $5.46 \pm 4.89$ & $12.6 \pm 9.91$ & $20.2 \pm 8.08$ & $54.5 \pm 33.7$ \\
\hline Liver (ng/g) & $11.7 \pm 6.80$ & $127 \pm 37.8^{* * *}$ & $150 \pm 32.5^{* *}$ & $215 \pm 72.9^{* * *}$ \\
\hline Spleen (ng/g) & $13.7 \pm 7.55$ & $22.6 \pm 5.72$ & $42.7 \pm 20.4$ & $79.5 \pm 58.6$ \\
\hline Kidney (ng/g) & $3.08 \pm 4.22$ & $32.7 \pm 11.1^{* *}$ & $37.6 \pm 15.3^{* *}$ & $70.1 \pm 24.2^{* *}$ \\
\hline Whole blood (ng/g) & $0.00 \pm 0.01$ & $0.04 \pm 0.04$ & $0.22 \pm 0.34$ & $0.18 \pm 0.18$ \\
\hline
\end{tabular}

${ }^{a}$ Values are expressed as means \pm SD.

${ }^{* * * *}$ Significantly different from vehicle control at $p<0.05$ and $p<0.01$.

\section{DISCUSSION}

While utilization of neodymium is increasing in various industrial fields due to its unique properties, there are growing concerns about potential risks to human health and the environment due to insufficient toxicological data and an absence of related regulations. Consequently, toxicological data are required for risk/hazard assessment of neodymium, in order to prevent possible adverse effects to humans and the environment. In a previous research, it was thought that men might be more sensitive to REEs than women, as the mean concentrations of all 15 REEs were much higher in the hair of men than in women from mining areas (22). Additionally, a higher toxicity was observed both in vivo and in vitro with a decrease in the particle size of lanthanum oxide $\left(\mathrm{La}_{2} \mathrm{O}_{3}\right)(21)$. Therefore, the present study was performed to determine the potential toxicity and target organs of the nano-sized $\mathrm{Nd}_{2} \mathrm{O}_{3}$ after a 28-day repeated inhalation exposure in male Sprague-Dawley rats, and to provide reference data for further inhalation toxicity studies.

In the present study, local changes related to the lung were the main observations, including changes in organ weight, histopathology, and BALF after 28-day repeated inhalation exposure to nano-sized $\mathrm{Nd}_{2} \mathrm{O}_{3}$. However, systemic changes including abnormal clinical signs and changes in body weight were not observed. These results were similar to those obtained in a previous inhalation toxicity study using nano-sized $\mathrm{La}_{2} \mathrm{O}_{3}$ in rats (23).

With regards to organ weight, lung weight increased significantly in all treatment groups due to exposure to the nano-sized $\mathrm{Nd}_{2} \mathrm{O}_{3}$. After the 28-day recovery period, this change increased further in the $2.5 \mathrm{mg} / \mathrm{m}^{3}$ and higher dose treatment groups, and decreased in the $0.5 \mathrm{mg} / \mathrm{m}^{3}$ treatment group; therefore, a clear dose-dependent relationship was observed. Increased lung weight was considered to be due to pulmonary alveolar proteinosis (PAP) in histopathology, as the occurrence and time-course change of PAP for each dose were consistent with the changes in lung weight, and this lesion induced an abnormal accumulation of lipoproteinaceous material within the alveoli (24). Trapnell et al. (25) reported that secondary PAP was associated with a disorder of surfactant homeostasis due to a functional impairment or reduced numbers of macrophages, the clinical significance of which manifested in increased susceptibility to pulmonary infections. It was also reported that PAP was caused by inhalation of inorganic dust or fumes in humans and animals $(26,27)$. In connection to this, the BALF had an opaque, milky appearance and abnormal alveolar macrophages were observed in the BALF of all treatment groups. It is well known that milky fluid and foamy alveolar macrophages are commonly observed in BALF of patients with PAP $(28,29)$. In the $0.5 \mathrm{mg} / \mathrm{m}^{3}$ treatment group, the severity of PAP was low, below mild grade, and recovery potential was observed with related changes in organ weight and BALF.

With regards to histopathology results, hyperplasia/hypertrophy of alveolar type II cells in the lungs of the 0.5 and 10 $\mathrm{mg} / \mathrm{m}^{3}$ treatment groups was not considered toxicologically significant, as the observed frequency and severity of the lesion were low. Although alveolar/perivascular infiltration of inflammatory cells was observed in all study groups, it was considered that the lesion observed at doses of 2.5 and 
$10 \mathrm{mg} / \mathrm{m}^{3}$ was a treatment-related effect, considering the observed frequency and severity of the lesion, and the apparent change above moderate grade at a dose of $10 \mathrm{mg} /$ $\mathrm{m}^{3}$ was toxicologically significant. In connection with the infiltration of inflammatory cells and PAP, proinflammatory cytokines (IL-6, TNF- $\alpha$ ), cellular damage indicators (albumin, LDH), and total cell counts, including macrophages and neutrophils, increased more sensitively in BALF obtained from all treatment groups after the 28-day exposure period. These changes nearly recovered to the control level in the $0.5 \mathrm{mg} / \mathrm{m}^{3}$ treatment group, but deteriorated in the $10 \mathrm{mg} / \mathrm{m}^{3}$ treatment group after 28-day recovery period. It was considered that a series of changes concerning inflammatory response, accompanied by aggregation of macrophages in the lung and hilar lymph node, was caused by an immune response to non-destroyed foreign metals, and prolonged and deteriorated changes were due to slow elimination of these materials in the $10 \mathrm{mg} / \mathrm{m}^{3}$ treatment group, resulting in apparent cell damage and alveolar macrophage impairment in the lung.

Results of neodymium levels in major organs were consistent with local changes observed in organ weight, histopathology, and BALF. Most of the inhaled neodymium was deposited in the lung after the 28-day exposure period, and the concentration significantly increased in a dose-dependent manner. High neodymium concentrations were maintained even after the 28-day recovery period. The percentage decrease was 21,34 , and $34 \%$ in the $0.5,2.5$, and $10 \mathrm{mg} / \mathrm{m}^{3}$ treatment groups, respectively, after the 28-day recovery period, and the half-life of nano-sized $\mathrm{Nd}_{2} \mathrm{O}_{3}$ in the lung was calculated to be 83,47 , and 47 days, respectively. In previous studies, the half-life of nano-sized cerium oxide $\left(\mathrm{CeO}_{2}\right)$ and $\mathrm{La}_{2} \mathrm{O}_{3}$ in the lung was calculated as 40 and 55 days, respectively, in rats exposed to $0.5 \mathrm{mg} / \mathrm{m}^{3}$ for 4 weeks $(20,23)$. Therefore, it was thought that nano-sized $\mathrm{Nd}_{2} \mathrm{O}_{3}$ may require a longer elimination time than nano-sized $\mathrm{CeO}_{2}$ and $\mathrm{La}_{2} \mathrm{O}_{3}$ under the same conditions. Neodymium in blood was detected at low levels with no dose-dependent relationship, which corresponded with the results of previous studies using $\mathrm{La}_{2} \mathrm{O}_{3}(21,23)$. Therefore, longer studies, or additional studies on kinetics, should be considered to identify a clear dose-dependent relationship and to elucidate this result.

With regards to oxidative stress analysis, GSH in the lung tissue decreased significantly in the 2.5 or/and $10 \mathrm{mg} / \mathrm{m}^{3}$ treatment groups after the exposure and recovery periods, but catalase activity did not change significantly. Many mechanism studies have reported that oxidative stress was induced in animal and plant models by exposure to cerium, lanthanum, and neodymium (16,30-32). Specifically, Peng et al. (33) reported two waves of lung injury by particle size that were mainly induced by macrophage phagocytosis in the case of $313 \mathrm{~nm}$ cerium agglomerates, and by earlier macrophage phagocytosis and later reactive oxygen species (ROS) generation in the case of $1,731 \mathrm{~nm}$ cerium agglomer- ates. Therefore, lung injury in the present study was mainly caused by phagocytosis rather than by ROS generation, considering the particle sizes of nano-sized $\mathrm{Nd}_{2} \mathrm{O}_{3}(270 \sim$ $670 \mathrm{~nm}$ ), and the alveolar macrophage issues in BALF. However, further studies are needed to elucidate the exact mechanism of oxidative stress.

In conclusion, 28-day repeated inhalation exposure to nano-sized $\mathrm{Nd}_{2} \mathrm{O}_{3}$ in rats resulted in increased lung weight, histopathological changes such as PAP and infiltration of inflammatory cells $\left(2.5\right.$ and $10 \mathrm{mg} / \mathrm{m}^{3}$ dose groups only), and increased counts of total cells, macrophages, and neutrophils, and levels of IL-6, TNF- $\alpha$, albumin, and LDH in BALF for all treatment groups. However, the severity of PAP was low for the $0.5 \mathrm{mg} / \mathrm{m}^{3}$ group. After a 4-week recovery period, these changes generally recovered for the $0.5 \mathrm{mg} / \mathrm{m}^{3}$ group, but deteriorated in the $10 \mathrm{mg} / \mathrm{m}^{3}$ group. Under the present experimental conditions, in male rats, the LOAEC of nano-sized $\mathrm{Nd}_{2} \mathrm{O}_{3}$ was determined to be $0.5 \mathrm{mg} / \mathrm{m}^{3}$, and the target organ was determined to be the lung. More precise toxicity assessment is required for further studies, considering the changes observed due to exposure at $10 \mathrm{mg} / \mathrm{m}^{3}$.

\section{CONFLICT OF INTEREST}

The authors declare that there are no conflicts of interest.

Received March 7, 2017; Revised May 2, 2017; Accepted May 10, 2017

\section{REFERENCES}

1. Hirano, S. and Suzuki, K.T. (1996) Exposure, metabolism, and toxicity of rare earths and related compounds. Environ. Health Perspect., 104 Suppl 1, 85-95.

2. Rim, K.T., Koo, K.H. and Park, J.S. (2013) Toxicological evaluations of rare earths and their health impacts to workers: a literature review. Saf. Health Work, 4, 12-26.

3. Raymond, D.H. and David, R.J. (2015) Rare earth metals in Hamilton \& Hardy's Industrial Toxicology (Raymond, D.H., Marie, M.B. and Giffe, T.J. Ed.). John Wiley \& Sons, Hoboken, NJ, pp. 199-204.

4. Haque, N., Hughes, A., Lim, S. and Vernon, C. (2014) Rare earth elements: overview of mining, mineralogy, uses, sustainability and environmental impact. Resources, 3, 614-635.

5. Sabbioni, E., Pietra, R., Gaglione, P., Vocaturo, G., Colombo, F., Zanoni, M. and Rodi, F. (1982) Long-term occupational risk of rare-earth pneumoconiosis. A case report as investigated by neutron activation analysis. Sci. Total Environ., 26, 19-32.

6. Vocaturo, G., Colombo, F., Zanoni, M., Rodi, F., Sabbioni, E. and Pietra, R. (1983) Human exposure to heavy metals. Rare earth pneumoconiosis in occupational workers. Chest, 83, 780-783.

7. Pietra, R., Sabbioni, E., Orvini, E., Vocaturo, G., Colombo, F., Zanoni, M. and Rodi, F. (1984) Occupational risk to rare earths. Inorganica Chim. Acta, 94, 143-144.

8. Sulotto, F., Romano, C., Berra, A., Botta, G.C., Rubino, G.F., 
Sabbioni, E. and Pietra, R. (1986) Rare-earth pneumoconiosis: a new case. Am. J. Ind. Med., 9, 567-575.

9. Vogt, P., Spycher, M.A. and Rüttner, J.R. (1986) Pneumoconiosis caused by "rare earths" (cer-pneumoconiosis). Schweiz. Med. Wochenschr., 116, 1303-1308.

10. Waring, P.M. and Watling, R.J. (1990) Rare earth deposits in a deceased movie projectionist. A new case of rare earth pneumoconiosis? Med. J. Aust., 153, 726-730.

11. Porru, S., Placidi, D., Quarta, C., Sabbioni, E., Pietra, R. and Fortaner, S. (2001) The potencial role of rare earths in the pathogenesis of interstitial lung disease: a case report of movie projectionist as investigated by neutron activation analysis. J. Trace Elem. Med. Biol., 14, 232-236.

12. Rim, K.T. (2017) Trends in Occupational Toxicology of Rare Earth Elements in Rare Earth Elements in Human and Environmental Health (Pagano, G. Ed.). Pan Stanford Publishing, Singapore, pp. 11-46.

13. European Chemicals Agency (ECHA) (2016) ECHA database on registered substances. Available from: http://echa. europa.eu/information-on-chemicals/registered-substances/.

14. Cheng, J., Cheng, Z., Hu, R., Cui, Y., Cai, J., Li, N., Gui, S., Sang, X., Sun, Q., Wang, L. and Hong, F. (2014) Immune dysfunction and liver damage of mice following exposure to lanthanoids. Environ. Toxicol., 29, 64-73.

15. Cheng, J., Li, N., Cai, J., Cheng, Z., Hu, R., Zhang, Q., Wan, F., Sun, Q., Gui, S., Sang, X., Wang, L. and Hong, F. (2012) Organ histopathological changes and its function damage in mice following long-term exposure to lanthanides chloride. Biol. Trace Elem. Res., 145, 361-368.

16. Huang, P., Li, J., Zhang, S., Chen, C., Han, Y., Liu, N., Xiao, Y., Wang, H., Zhang, M., Yu, Q., Liu, Y. and Wang, W. (2011) Effects of lanthanum, cerium, and neodymium on the nuclei and mitochondria of hepatocytes: accumulation and oxidative damage. Environ. Toxicol. Pharmacol., 31, 25-32.

17. Zhao, H., Cheng, Z., Hu, R., Chen, J., Hong, M., Zhou, M., Gong, X., Wang, L. and Hong, F. (2011) Oxidative injury in the brain of mice caused by lanthanid. Biol. Trace Elem. Res., 142, 174-189.

18. Palmer, R.J., Butenhoff, J.L. and Stevens, J.B. (1987) Cytotoxicity of the rare earth metals cerium, lanthanum, and neodymium in vitro: comparisons with cadmium in a pulmonary macrophage primary culture system. Environ. Res., 43, 142156.

19. Takaya, M., Toya, T., Takata, A., Otaki, N., Yoshida, K. and Kohyama, N. (2005) Biological effects of rare earth oxides to respiratory organs. J. Aerosol Res., 20, 207-212.

20. Keller, J., Wohlleben, W., Ma-Hock, L., Strauss, V., Gröters, S., Küttler, K., Wiench, K., Herden, C., Oberdörster, G., van Ravenzwaay, B. and Landsiedel, R. (2014) Time course of lung retention and toxicity of inhaled particles: short-term exposure to nano-Ceria. Arch. Toxicol., 88, 2033-2059.

21. Lim, C.H. (2015) Toxicity of two different sized lanthanum oxides in cultured cells and sprague-dawley rats. Toxicol. Res., 31, 181-189.

22. Wei, B., Li, Y., Li, H., Yu, J., Ye, B. and Liang, T. (2013) Rare earth elements in human hair from a mining area of China. Ecotoxicol. Environ. Saf., 96, 118-123.

23. Shin, S.H., Lim, C.H., Kim, Y.S., Lee, Y.H., Kim, S.H. and Kim, J.C. (2017) Twenty-eight-day repeated inhalation toxicity study of nano-sized lanthanum oxide in male sprague-dawley rats. Environ. Toxicol., 32, 1226-1240.

24. Rosen, S.H., Castleman, B. and Liebow, A.A. (1958) Pulmonary alveolar proteinosis. N. Engl. J. Med., 258, 1123-1142.

25. Trapnell, B.C., Whitsett, J.A. and Nakata, K. (2003) Pulmonary alveolar proteinosis. N. Engl. J. Med., 349, 2527-2539.

26. Keller, C.A., Frost, A., Cagle, P.T. and Abraham, J.L. (1995) Pulmonary alveolar proteinosis in a painter with elevated pulmonary concentrations of titanium. Chest, 108, 277-280.

27. Serita, F., Kyono, H. and Seki, Y. (1999) Pulmonary clearance and lesions in rats after a single inhalation of ultrafine metallic nickel at dose levels comparable to the threshold limit value. Ind. Health, 37, 353-363.

28. Danel, C., Israel-Biet, D., Costabel, U., Rossi, G.A. and Wallaert, B. (1990) The clinical role of BAL in alveolar proteinosis. Eur. Respir. J., 3, 950-1, 961-9.

29. Shah, P.L., Hansell, D., Lawson, P.R., Reid, K.B. and Morgan, C. (2000) Pulmonary alveolar proteinosis: clinical aspects and current concepts on pathogenesis. Thorax, 55, 67-77.

30. Zhao, L., Peng, B., Hernandez-Viezcas, J.A., Rico, C., Sun, Y., Peralta-Videa, J.R., Tang, X., Niu, G., Jin, L., VarelaRamirez, A., Zhang, J.Y. and Gardea-Torresdey, J.L. (2012) Stress response and tolerance of Zea mays to $\mathrm{CeO}_{2}$ nanoparticles: Cross talk among $\mathrm{H}_{2} \mathrm{O}_{2}$, heat shock protein, and lipid peroxidation. ACS Nano, 6, 9615-9622.

31. Hong, J., Pan, X., Zhao, X., Yu, X., Sang, X., Sheng, L., Wang, X., Gui, S., Sun, Q., Wang, L. and Hong, F. (2015) Molecular mechanism of oxidative damage of lung in mice following exposure to lanthanum chloride. Environ. Toxicol., 30, 357-365.

32. Morimoto, Y., Izumi, H., Yoshiura, Y., Tomonaga, T., Oyabu, T., Myojo, T., Kawai, K., Yatera, K., Shimada, M., Kubo, M., Yamamoto, K., Kitajima, S., Kuroda, E., Kawaguchi, K. and Sasaki, T. (2015) Pulmonary toxicity of well-dispersed cerium oxide nanoparticles following intratracheal instillation and inhalation. J. Nanopart. Res., 17, 442.

33. Peng, L., He, X., Zhang, P., Zhang, J., Li, Y., Zhang, J., Ma, Y., Ding, Y., Wu, Z., Chai, Z. and Zhang, Z. (2014) Comparative pulmonary toxicity of two ceria nanoparticles with the same primary size. Int. J. Mol. Sci., 15, 6072-6085. 